\title{
CONICAL LIMIT POINTS AND THE CANNON-THURSTON MAP
}

\author{
WOOJIN JEON, ILYA KAPOVICH, CHRISTOPHER LEININGER, \\ AND KEN'ICHI OHSHIKA
}

\begin{abstract}
Let $G$ be a non-elementary word-hyperbolic group acting as a convergence group on a compact metrizable space $Z$ so that there exists a continuous $G$-equivariant map $i: \partial G \rightarrow Z$, which we call a Cannon-Thurston map. We obtain two characterizations (a dynamical one and a geometric one) of conical limit points in $Z$ in terms of their pre-images under the CannonThurston map $i$. As an application we prove, under the extra assumption that the action of $G$ on $Z$ has no accidental parabolics, that if the map $i$ is not injective, then there exists a non-conical limit point $z \in Z$ with $\left|i^{-1}(z)\right|=1$. This result applies to most natural contexts where the Cannon-Thurston map is known to exist, including subgroups of word-hyperbolic groups and Kleinian representations of surface groups. As another application, we prove that if $G$ is a non-elementary torsion-free word-hyperbolic group, then there exists $x \in \partial G$ such that $x$ is not a "controlled concentration point" for the action of $G$ on $\partial G$.
\end{abstract}

\section{INTRODUCTION}

Let $G$ be a Kleinian group, that is, a discrete subgroup of the isometry group of hyperbolic space $G \leq \operatorname{Isom}^{+}\left(\mathbb{H}^{n}\right)$. In [6], Beardon and Maskit defined the notion of a conical limit point (also called a point of approximation or radial limit point), and used this to provide an alternative characterization of geometric finiteness. Gehring and Martin 42 abstracted the notion of Kleinian group to that of a convergence group acting on $\mathbb{S}^{n-1}$, which was then further generalized, for example by Tukia in [86], to actions on more general compact metric spaces (see Definition 2.1). This generalization includes, for example, the action of a discrete group of isometries of a proper, Gromov-hyperbolic, metric space on its boundary at infinity; see [36, 86. Conical limit points can be defined in this level of generality (see Definition 2.4), and play a key role in the convergence group characterization of word-hyperbolic groups by Bowditch [16, of relatively hyperbolic groups by Yaman [88, and of quasiconvex subgroups of word-hyperbolic groups by Swenson [83], and arise in numerous other results in topology, geometry, and dynamics; see, for example,

Received by the editors May 7, 2015 and, in revised form, January 29, 2016

2010 Mathematics Subject Classification. Primary 20F65; Secondary 30F40, 57M60, 37Exx, $37 \mathrm{Fxx}$.

Key words and phrases. Convergence groups, Cannon-Thurston map, conical limit points, Kleinian groups.

The second author was partially supported by Collaboration Grant no. 279836 from the Simons Foundation and by NSF grant DMS-1405146. The third author was partially supported by NSF grants DMS-1207183 and DMS-1510034. The last author was partially supported by JSPS Grants-in-Aid 70183225. 
[2, 26, 34, 37, 38, 58, 59] for other results involving convergence groups and conical limit points.

In their 1984 preprint, published in 2007 [25], Cannon and Thurston proved the following remarkable result. If $M$ is a closed hyperbolic 3-manifold fibering over a circle with fiber a closed surface $\Sigma$, then the inclusion $\mathbb{H}^{2}=\widetilde{\Sigma} \subset \widetilde{M}=\mathbb{H}^{3}$ extends to a continuous surjective map $\mathbb{S}^{1}=\partial \mathbb{H}^{2} \rightarrow \partial \mathbb{H}^{3}=\mathbb{S}^{2}$, equivariant with respect to $\pi_{1}(\Sigma)$ which is acting as a convergence group on both. Based on this we make the following general, abstract definition.

Definition 1.1. When $G$ is a word-hyperbolic group acting as a convergence group on a compact metrizable space $Z$, a map $i: \partial G \rightarrow Z$ is called a Cannon-Thurston map if $i$ is continuous and $G$-equivariant.

Under some mild assumptions, it is known that if a Cannon-Thurston map $i: \partial G \rightarrow Z$ exists, then it is unique; see Proposition 2.11 below. Of particular interest is the case that a non-elementary word-hyperbolic group $G$ acts on a proper, Gromov hyperbolic, geodesic metric space $Y$, properly discontinuously by isometries, and without accidental parabolics (see Definition 2.8). In this case, if there exists a Cannon-Thurston map $i: \partial G \rightarrow \partial Y$, then it is known to be unique and to extend to a $G$-equivariant continuous map $G \cup \partial G \rightarrow Y \cup \partial Y$ (see Proposition 2.12). A special subcase of interest is when $G_{1}, G_{2}$ are non-elementary word-hyperbolic groups, with $G_{1} \leq G_{2}$ acting on (the Cayley graph of) $G_{2}$ by restriction of the left action of $G_{2}$ on itself. Here a Cannon-Thurston map is classically defined as a continuous extension $G_{1} \cup \partial G_{1} \rightarrow G_{2} \cup \partial G_{2}$ of the inclusion of $G_{1} \rightarrow G_{2}$. By Proposition 2.12, the existence of such a map is equivalent to the existence of a Cannon-Thurston map in the sense of Definition 1.1 for the induced action of $G_{1}$ on $\partial G_{2}$. Quasi-isometrically embedded subgroups $G_{1} \leq G_{2}$ of word-hyperbolic groups provide examples where Cannon-Thurston maps exist. However, CannonThurston's original result [25] described above implies that for the word-hyperbolic groups $G_{1}=\pi_{1}(\Sigma) \leq \pi_{1}(M)=G_{2}$, there is a Cannon-Thurston map $\partial G_{1} \rightarrow \partial G_{2}$, but here $G_{1}$ is exponentially distorted in $G_{2}$. Subsequent work of Mitra 68, 69] showed that there are many other interesting situations where $G_{1}$ is not quasiconvex in $G_{2}$ but where the Cannon-Thurston map nevertheless does exist (see also [5]). On the other hand, a recent remarkable result of Baker and Riley 4 proves that there exists a word-hyperbolic group $G_{2}$ and a word-hyperbolic (in fact, non-abelian free) subgroup $G_{1} \leq G_{2}$ such that the Cannon-Thurston map $i: \partial G_{1} \rightarrow \partial G_{2}$ does not exist.

Generalizing the Cannon-Thurston example from 25] in another direction, one can consider other actions of $G=\pi_{1}(\Sigma)$, the fundamental group of a closed, orientable surface of genus at least 2, acting properly discontinuously by isometries on $\mathbb{H}^{3}$, i.e. as a classical Kleinian surface group. The first partial results beyond those in 25] about the existence of Cannon-Thurston maps for such actions of $G$ on $\mathbb{H}^{3}$ are due to Minsky [65]. Extending beyond the case $G=\pi_{1}(\Sigma)$, there have been numerous results on the existence of Cannon-Thurston maps of various types (not necessarily fitting into Definition 1.1), especially for Kleinian groups [18, 19, 35, 60, 64, 70, 72, 75, 81. Recently, Mj 77] has shown that for any properly discontinuous action on $\mathbb{H}^{3}$ without accidental parabolics, there exists a CannonThurston map, using the theory of model manifolds which were developed by Minsky. There are extensions of the Cannon-Thurston maps also for subgroups of mapping class groups [62], and in other related contexts [39, 41]. 
Mj has also shown 71 that in the case of classical Kleinian surface groups without parabolics, the non-injective points of a Cannon-Thurston map are exactly the endpoints of the lifts of the ending laminations to the domains of discontinuity. This characterization of non-injective points of Cannon-Thurston maps has some applications: for instance, the first and the fourth authors have used this to prove the measurable rigidity for Kleinian groups (see [46]), which is a generalization of the results by Sullivan 82 and Tukia 84 . Also, using the same kind of characterization for free classical Kleinian groups, Jeon-Kim-Ohshika-Lecuire 45] gave a criterion for points on the boundary of the Schottky space to be primitive stable.

Another reason to be interested in understanding injective points of CannonThurston maps comes from the study of dynamics and geometry of fully irreducible elements of $\operatorname{Out}\left(F_{N}\right)$. If $\varphi \in \operatorname{Out}\left(F_{N}\right)$ is an atoroidal fully irreducible element, then the mapping torus group $G_{\varphi}=F_{N} \rtimes_{\varphi} \mathbb{Z}$ is word-hyperbolic and the CannonThurston map $i: \partial F_{N} \rightarrow \partial G_{\varphi}$ exists by the result of [68. In this case, if $T_{ \pm}$are the "attracting" and "repelling" $\mathbb{R}$-trees for $\varphi$, there are associated $\mathcal{Q}$-maps (defined in [32])

$$
\mathcal{Q}_{+}: \partial F_{N} \rightarrow \widehat{T}_{+}=\overline{T_{+}} \cup \partial T_{+}
$$

and

$$
\mathcal{Q}_{-}: \partial F_{N} \rightarrow \widehat{T}_{-}=\overline{T_{-}} \cup \partial T_{-}
$$

(here $\overline{T_{ \pm}}$denotes the metric completion of $T_{ \pm}$). These maps play an important role in the index theory of free group automorphisms, particularly for the notion of $\mathcal{Q}$-index; see $28,30,32$. It is shown in 53 that a point $x \in \partial F_{N}$ is injective for the Cannon-Thurston map $i$ if and only if $x$ is injective for both $\mathcal{Q}_{+}$and $\mathcal{Q}_{-}$.

There are a number of results in the literature which prove in various situations where the Cannon-Thurston map exists that every conical limit point is "injective", that is, has exactly one pre-image under the Cannon-Thurston map; see, for example, 39, 56, 61]. We discuss some of these facts in more detail after the statement of Theorem B below. These results naturally raise the question of whether the converse holds, that is, whether a point with exactly one pre-image under the Cannon-Thurston map must be a conical limit point. (The only result in the literature dealing with this converse direction is Theorem 8.6 in [56, which incorrectly claims that every "injective" limit point is conical in the original setting of a closed hyperbolic 3-manifold fibering over a circle.) We show in Theorem C below that the converse statement fails in great generality and prove that, under rather mild assumptions, if a Cannon-Thurston map exists and is not injective, then there always exists a non-conical limit point with exactly one pre-image under the Cannon-Thurston map.

In this paper, given a non-elementary convergence action of a word-hyperbolic group $G$ on a compact metrizable space $Z$, such that the Cannon-Thurston map $i: \partial G \rightarrow Z$ exists, we give two characterizations (a dynamical one and a geometric one) of conical limit points $z \in Z$ in terms of their pre-images under the map $i$.

To state these characterizations we need to introduce some definitions. Under the above assumptions, denote $L_{i}=\{(x, y) \mid x, y \in \partial G, i(x)=i(y)$, and $x \neq y\}$. We say that a point $x \in \partial G$ is asymptotic to $L_{i}$ if for every conical sequence $\left\{g_{n}\right\}_{n=1}^{\infty}$ for $x$ with pole pair $\left(x_{-}, x_{+}\right)$, we have $\left(x_{-}, x_{+}\right) \in L_{i}$, that is, $i\left(x_{-}\right)=i\left(x_{+}\right)$. (See Definition 2.4 below for the notions of a conical sequence and pole pair.) 
The following result provides a dynamical characterization of conical limit points in $Z$ :

Theorem A. Suppose $G$ is word-hyperbolic and acts on the compact, metrizable space $Z$ as a non-elementary convergence group, and suppose $i: \partial G \rightarrow Z$ is a Cannon-Thurston map. Let $z \in i(\partial G)$. Then:

(1) The point $z \in Z$ is not a conical limit point for the action of $G$ on $Z$ if and only if some point $x \in i^{-1}(z)$ is asymptotic to $L_{i}$.

(2) If $\left|i^{-1}(z)\right|>1$, then any $x \in i^{-1}(z)$ is asymptotic to $L_{i}$, and hence $z$ is non-conical.

We also provide a geometric counterpart of Theorem A

Theorem B. Let $G$ be a word-hyperbolic group and let $Z$ be a compact metrizable space equipped with a non-elementary convergence action of $G$ such that the Cannon-Thurston map $i: \partial G \rightarrow Z$ exists and such that $i$ is not injective. Let $X$ be a $\delta$-hyperbolic (where $\delta \geq 1$ ) proper geodesic metric space equipped with a properly discontinuous cocompact isometric action of $G$ (so that $\partial G$ is naturally identified with $\partial X)$.

Let $x \in \partial G$, let $z=i(x) \in Z$ and let $\rho$ be a geodesic ray in $X$ limiting to $x$.

Then the following are equivalent:

(1) The point $z$ is a conical limit point for the action of $G$ on $Z$.

(2) There exist a geodesic segment $\tau=[a, b]$ in $X$ of length $\geq 100 \delta$ and an infinite sequence of distinct elements $g_{n} \in G$ such that the $20 \delta$-truncation $\tau^{\prime}$ of $\tau$ is not a coarse $X$-leaf segment of $L_{i}$ and such that for each $n \geq 1$ the segment $g_{n} \tau$ is contained in a $6 \delta$-neighborhood of $\rho$, and that $\lim _{n \rightarrow \infty} g_{n} a=$ $\lim _{n \rightarrow \infty} g_{n} b=x$.

See the definition of a "coarse leaf segment" and of other relevant terms in Section 5 .

Theorem $\mathrm{A}$ is used in the proof of Theorem $\mathrm{C}$, as discussed in more detail below. Theorem $\mathrm{B}$ is used as a key ingredient in the proof of Theorem 6.6 of 33 .

Theorems $\mathrm{A}$ and $\mathrm{B}$ are partially motived by the result of M. Kapovich 56, who proved that in the setting of Cannon and Thurston's original construction 25] of a Cannon-Thurston map $i: \partial G \rightarrow \mathbb{S}^{2}$, for $G=\pi_{1}(\Sigma)$, if $z \in \partial \mathbb{S}^{2}=\partial G$ has $\left|i^{-1}(z)\right| \geq 2$, then $z$ is not a conical limit point for the action of $G$ on $\mathbb{S}^{2}$. This result was extended by Leininger, Long and Reid [61, who proved that the same result for any doubly degenerate Kleinian representation (where $i$ exists from [77]), and later by Gerasimov [39, for arbitrary " $\times$-actions". In fact, part (2) of Theorem A follows from a general result of Gerasimov [39, Proposition 7.5.2] about conical limit points for $\times$-actions. Gerasimov also explained to us how one can derive part (1) of Theorem $\mathrm{A}$ from the results of 39 using a result of Bowditch. We provide a short direct proof of Theorem $\mathrm{A}$ here.

It is known by results of Swenson 83 , and $\mathrm{Mj}$ [76] that in the geometric context, where the Cannon-Thurston map $i: \partial G \rightarrow \partial Y$ arises from a properly discontinuous isometric action of a word-hyperbolic $G$ on a proper Gromov-hyperbolic space $Y$, if $i: \partial G \rightarrow \partial Y$ is injective, then the orbit-map $G \rightarrow Y$ is a quasi-isometric embedding; see Proposition 2.13 below for a precise statement. In this case every limit point 
$z \in \partial Y$ is conical and has exactly one pre-image under $i$. Therefore Theorem $\mathrm{A}$ implies:

Corollary 1.2. Let $G$ be a non-elementary word-hyperbolic group equipped with a properly discontinuous isometric action on a proper geodesic Gromov-hyperbolic space $Y$ without accidental parabolics. Suppose that the Cannon-Thurston map $i: \partial G \rightarrow \partial Y$ exists.

Then there exists $z \in i(\partial G)$ such that $z$ is a non-conical limit point for the action of $G$ on $\partial Y$ if and only if $i$ is not injective.

Proof. If $i$ is not injective and $x_{1}, x_{2} \in \partial G$ are points such that $x_{1} \neq x_{2}$ and that $i\left(x_{1}\right)=i\left(x_{2}\right)$, then, by part (2) of Theorem $z=i\left(x_{1}\right)=i\left(x_{2}\right)$ is not conical. If the map $i$ is injective, then, since $\partial G$ and $i(\partial G) \subseteq \partial Y$ are compact and Hausdorff, the map $i$ is a $G$-equivariant homeomorphism between $\partial G$ and $i(\partial G)$. Since $G$ is hyperbolic, every point of $\partial G$ is conical for the action of $G$ on $\partial G$; see 86 . Therefore every $z \in i(\partial G)$ is conical for the action of $G$ on $i(\partial G)$ and hence, by Lemma 2.6. also for the action of $G$ on $\partial Y$.

The main result of this paper is:

Theorem C. Suppose a word-hyperbolic group $G$ acts on a compact metrizable space $Z$ as a non-elementary convergence group without accidental parabolics, and suppose that there exists a non-injective Cannon-Thurston map $i: \partial G \rightarrow Z$. Then there exists a non-conical limit point $z \in Z$ with $\left|i^{-1}(z)\right|=1$.

Theorem C] applies whenever $G_{1}$ is a non-elementary non-quasiconvex wordhyperbolic subgroup of a word-hyperbolic group $G_{2}$ such that the Cannon-Thurston map $\partial G_{1} \rightarrow \partial G_{2}$ exists. Similarly, Theorem C applies whenever $\Sigma$ is a closed hyperbolic surface and $\pi_{1}(\Sigma)$ is equipped with a properly discontinuous isometric action on $\mathbb{H}^{3}$ without accidental parabolics, assuming that the Cannon-Thurston map $\mathbb{S}^{1}=\partial \pi_{1}(\Sigma) \rightarrow \partial \mathbb{H}^{3}=\mathbb{S}^{2}$ exists and is non-injective.

Brian Bowditch (private communication) showed us another argument for obtaining the conclusion of Theorem $\mathrm{C}$ for a large class of Kleinian groups, including the original case of a closed hyperbolic 3-manifold fibering over a circle. His argument is different from the proof presented in this paper and relies on the Kleinian groups and 3-manifold methods.

Another application of our results concerns "controlled concentration points". Originally, the notion of a controlled concentration point was defined for a properly discontinuous isometric action of a torsion-free group $G$ on $\mathbb{H}^{n}$. A point $x$ in $\partial \mathbb{H}^{n}$ is called a controlled concentration point of $G$ when $x$ has a neighborhood $V$ such that for any neighborhood $U$ of $x$ there is $g \in G$ with $g U \subset V$ and $x \in g(V)$. This is equivalent to saying that there is a sequence of elements $\left(g_{n}\right)_{n \geq 1} \subset G$ such that $g_{n}(x) \rightarrow x$ and $\left(\left.g_{n}\right|_{\partial \mathbb{H}^{n} \backslash\{x\}}\right)$ converges locally uniformly to a constant map to some point $y \neq x$. Aebischer, Hong and McCullough 3 . showed that a limit point $x \in \partial \mathbb{H}^{n}$ is a controlled concentration point if and only if it is an endpoint of a lift of a recurrent geodesic ray in $M:=\mathbb{H}^{n} / G$. A geodesic ray $\alpha(t)$ in $M$ is called recurrent if for any $t_{0}$, there exists a sequence $\left\{t_{i}\right\}$ with $t_{i} \rightarrow \infty$ such that $\lim _{i \rightarrow \infty} \alpha^{\prime}\left(t_{i}\right)=\alpha^{\prime}\left(t_{0}\right)$ in the unit tangent bundle of $M$. They also showed there exist non-controlled concentration points in the limit set of a rank-2 Schottky group.

We generalize the notion of controlled concentration points to points at infinity of general word-hyperbolic groups by adopting the latter condition above as its 
definition; see Definition 7.4 below. As an application of Theorem C, we get the following existence theorem of non-controlled concentration points:

Theorem D. Let $G$ be a non-elementary torsion-free word-hyperbolic group. Then there exists $x \in \partial G$ which is not a controlled concentration point.

In Appendix $\mathrm{A}$ we discuss several specific situations where the Cannon-Thurston map $i: \partial G \rightarrow Z$ is known to exist and where a more detailed description of the lamination $L_{i}$ is known.

\section{Definitions AND BASIC FACTS}

\subsection{Convergence groups.}

Definition 2.1 (Convergence action). An action of a group $G$ on a compact metrizable space $Z$ by homeomorphisms is called a convergence action (in which case we also say that $G$ acts on $Z$ as a convergence group) if for any infinite sequence $\left(g_{n}\right)_{n \geq 1}$ of distinct elements of $G$ there exist $a, b \in Z$ and a subsequence $\left(g_{n_{k}}\right)_{k \geq 1}$ of $\left(g_{n}\right)$, called a convergence subsequence, such that the sequence of maps $\left\{\left.g_{n_{k}}\right|_{Z \backslash\{a\}}\right\}$ converges uniformly on compact subsets to the constant map $c_{b}: Z \backslash\{a\} \rightarrow Z$ sending $Z \backslash\{a\}$ to $b$. In this case we call $\left(a, b,\left(g_{n_{k}}\right)\right)$ the convergence subsequence data. The action is called elementary if either $G$ is finite or $G$ preserves a subset of $Z$ of cardinality $\leq 2$, and it is called non-elementary otherwise.

Note that if $G$ acts as a convergence group on $Z$ and if $Z^{\prime} \subseteq Z$ is a non-empty closed $G$-invariant subset, then the restricted action of $G$ on $Z^{\prime}$ is also a convergence action.

For a group $G$ acting on a set $Z$ and for $g \in G$ denote $F_{i x}(g):=\{z \in Z \mid g z=z\}$. The following is a basic fact about convergence groups; see [17, Lemma 3.1] and [86.

Proposition 2.2. Suppose $G$ acts as a convergence group on a compact metrizable space $Z$ and let $g \in G$. Then exactly one of the following occurs:

(1) The element $g$ has finite order in $G$; in this case $g$ is said to be elliptic.

(2) The element $g$ has infinite order in $G$ and the fixed set Fix ${ }_{Z}(g)$ consists of a single point; in this case $g$ is called parabolic.

(3) The element $g$ has infinite order in $G$ and the fixed set Fix $x_{Z}(g)$ consists of two distinct points; in this case $g$ is called loxodromic.

Moreover, for every $k \neq 0$ the elements $g$ and $g^{k}$ have the same type; also in cases (2) and (3) we have Fix $x_{Z}(g)=F i x_{Z}\left(g^{k}\right)$ and the group $\langle g\rangle$ acts properly discontinuously on $Z \backslash F i x_{Z}(g)$. Additionally, if $g \in G$ is loxodromic, then $\langle g\rangle$ acts properly discontinuously and cocompactly.

It is also known that if $g \in G$ is parabolic with a fixed point $a \in Z$, then for every $z \in Z$ we have $\lim _{n \rightarrow \infty} g^{n} z=\lim _{n \rightarrow-\infty} g^{n} z=a$. Also, if $g \in G$ is loxodromic, then we can write $\operatorname{Fix}_{Z}(g)=\left\{a_{-}, a_{+}\right\}$, and for every $z \in Z \backslash\left\{a_{+}\right\}$we have $\lim _{n \rightarrow \infty} g^{n} z=a_{-}$, and for every $z \in Z \backslash\left\{a_{-}\right\}$we have $\lim _{n \rightarrow-\infty} g^{n} z=a_{+}$, and these convergences are uniform on compact subsets of $Z \backslash\left\{a_{-}, a_{+}\right\}$.

Definition 2.3 (Limit set). If $G$ acts on $Z$ as a non-elementary convergence group, there exists a unique minimal non-empty closed $G$-invariant subset $\Lambda(G) \subseteq Z$ called 
the limit set of $G$ in $Z$. In this case $\Lambda(G)$ is perfect and hence $\Lambda(G)$ is infinite [86]. If $\Lambda(G)=Z$, then we say that the action of $G$ on $Z$ is minimal.

Definition 2.4 (Conical limit point). Let $G$ act on $Z$ as a convergence group. A point $z \in Z$ is called a conical limit point for the action of $G$ on $Z$ if there exist an infinite sequence $\left(g_{n}\right)_{n \geq 1}$ of distinct elements of $G$ and a pair of distinct points $z_{-}, z_{+} \in Z$ such that $\lim _{n \rightarrow \infty} g_{n} z=z_{+}$and that $\left(\left.g_{n}\right|_{Z \backslash\{z\}}\right)$ converges uniformly on compact subsets to the constant map $c_{z_{-}}: Z \backslash\{z\} \rightarrow Z$ sending $Z \backslash\{z\}$ to $z_{-}$. We call such a sequence $g_{n}$ a conical sequence for $z$, and the pair $\left(z_{-}, z_{+}\right)$the pole pair corresponding to $z$ and $\left(g_{n}\right)_{n \geq 1}$. If every point of $Z$ is a conical limit point, then the action is called a uniform convergence action. In particular, in this case the action is minimal.

Note that if $g \in G$ is loxodromic with $\operatorname{Fix}_{Z}(g)=\left\{a_{-}, a_{+}\right\}$, then both $a_{+}, a_{-}$ are conical limit points for the action of $G$ on $Z$, and one can use $\left(g^{n}\right)_{n \geq 1}$ as the conical sequence with pole pair $\left(a_{-}, a_{+}\right)$.

As usual, for $\delta \geq 0$, a $\delta$-hyperbolic space is a geodesic metric space $X$ such that for every geodesic triangle in $X$ each side of this triangle is contained in the $\delta$-neighborhood of the union of two other sides. A metric space $X$ is Gromovhyperbolic if there exists $\delta \geq 0$ such that $X$ is $\delta$-hyperbolic. A finitely generated group $G$ is called word-hyperbolic if for some (equivalently, any) finite generating set $\mathcal{S}$ of $G$, the Cayley graph of $G$ with respect to $\mathcal{S}$ is Gromov-hyperbolic. See [21] for basic background information about Gromov-hyperbolic spaces and word-hyperbolic groups; also see [49] for the background regarding boundaries of hyperbolic spaces and of word-hyperbolic groups.

Example 2.5. Let $G$ be an infinite word-hyperbolic group, and write $\partial G$ to denote the Gromov boundary. Then the action of $G$ on $\partial G$ is a uniform convergence action. In fact, according to a result of Bowditch [16], if the action of a group $G$ on a compact metrizable space $Z$ is a uniform convergence action, then $G$ is a word-hyperbolic and there is a $G$-equivariant homeomorphism between $\partial G$ and $Z$.

Lemma 2.6. Let a group $G$ act as a convergence group on $Z$, let $Z^{\prime} \subseteq Z$ be a non-empty infinite closed $G$-invariant subset, and let $z \in Z^{\prime}$. Then $z$ is a conical limit point for the action of $G$ on $Z$ if and only if $z$ is a conical limit point for the action of $G$ on $Z^{\prime}$.

Proof. The "only if" direction is obvious from the definition of a conical limit point. Thus suppose that $z \in Z^{\prime}$ is a conical limit point for the action of $G$ on $Z^{\prime}$. Let $\left(g_{n}\right)_{n}$ be a conical sequence for $z$ for the action of $G$ on $Z^{\prime}$ and let $\left(z_{-}, z_{+}\right)$be the corresponding pole pair (also for the action of $G$ on $Z^{\prime}$ ). Since $G$ acts on $Z$ as a convergence group, there exist convergence subsequence data $\left(a, b,\left(g_{n_{k}}\right)\right)$. By assumption $Z^{\prime}$ is infinite and hence for every $x \in Z^{\prime} \backslash\{a, z\}$ we have $\lim _{n \rightarrow \infty} g_{n} x=z_{-}$ and $\lim _{n \rightarrow \infty} g_{n} x=b$; it follows that $z_{-}=b$.

We claim that $a=z$. Indeed, suppose that $a \neq z$. Since $z \in Z \backslash\{a\}$, it follows that $\lim _{n \rightarrow \infty} g_{n} z=b=z_{-}$. On the other hand, by assumption about $\left(z_{-}, z_{+}\right)$being the pole pair for $z$ and $\left(g_{n}\right)_{n}$, it follows that $\lim _{n \rightarrow \infty} g_{n} z=z_{+}$. By definition $z_{-} \neq z_{+}$, which gives a contradiction. Thus indeed $a=z$. Hence $z$ is a conical limit point for the action of $G$ on $Z$, as claimed.

The following basic fact is well known; see, for example, [17. 
Proposition 2.7. Let $G$ be a word-hyperbolic group acting as a non-elementary convergence group on a compact metrizable space $Z$.

Then a $G$-limit point $z \in Z$ is a conical limit point for the action of $G$ on $Z$ if and only if for every point $s \in Z$ such that $s \neq z$ there exists an infinite sequence $g_{n} \in G$ of distinct elements of $G$ and points $s_{\infty}, z_{\infty} \in Z$ such that $s_{\infty} \neq z_{\infty}$ and such that $\lim _{n \rightarrow \infty} g_{n} z=z_{\infty}$ and $\lim _{n \rightarrow \infty} g_{n} s=s_{\infty}$.

Definition 2.8 (Accidental parabolic). Let $G$ be an infinite word-hyperbolic group acting as a non-elementary convergence group on a compact metrizable space $Z$. An accidental parabolic for this action is an infinite order element $g \in G$ such that $g$ acts parabolically on $Z$.

\subsection{Cannon-Thurston map.}

Definition 2.9 (Cannon-Thurston map). Let $G$ be a word-hyperbolic group acting as a non-elementary convergence group on a compact metrizable space $Z$. A map $i: \partial G \rightarrow Z$ is called a Cannon-Thurston map if $i$ is continuous and $G$-equivariant.

Lemma 2.10. Let $G$ be a word-hyperbolic group acting as a non-elementary convergence group on a compact metrizable space $Z$ and suppose $i: \partial G \rightarrow Z$ is a Cannon-Thurston map. Then:

(1) If $g$ acts as a loxodromic on $Z$, then the attracting and repelling fixed points in $\partial G$ of $g$, respectively, are sent by $i$ to the attracting and repelling fixed points of $g$ in $Z$, respectively.

(2) If $g$ is an accidental parabolic, then there is exactly one fixed point for $g$ on $Z$, which is the $i$-image of the two fixed points in $\partial G$.

Proof. Let $g \in G$ be an element of infinite order. Denote by $g^{\infty}$ and $g^{-\infty}$ the attracting and repelling points for $g$ in $\partial G$ respectively. Since $i$ is $G$-equivariant and the points $g^{ \pm \infty} \in \partial G$ are fixed by $g$, it follows that $i\left(\left\{g^{\infty}, g^{-\infty}\right\}\right) \subseteq F i x_{Z}(g)$. If $g$ is parabolic and $F_{Z} i x_{Z}(g)=\{a\}$, it follows that $i\left(g^{ \pm \infty}\right)=a$.

Suppose now that $g$ acts on $Z$ loxodromically. Since by assumption $G$ acts on $Z$ and hence on $i(\partial G)$ as a non-elementary convergence group, the set $i(\partial G)$ is infinite. Hence there exists $x \in \partial G$ such that $i(x) \notin F i x_{Z}(g)$ (and hence $x \notin\left\{g^{\infty}, g^{-\infty}\right\}$ ). If $g$ acts loxodromically on $Z$ with $\operatorname{Fix}_{Z}(g)=\left\{a_{+}, a_{-}\right\}$, then $\lim _{n \rightarrow \infty} g^{n} x=g^{\infty}$ and hence, by continuity and $g$-equivariance of $i, \lim _{n \rightarrow \infty} g^{n} i(x)=i\left(g^{\infty}\right)$. On the other hand, by definition of a loxodromic element, since $i(x) \neq a_{-}$we have $\lim _{n \rightarrow \infty} g^{n} i(x)=$ $a_{+}$. Thus $i\left(g^{\infty}\right)=a_{+}$. Replacing $g$ by $g^{-1}$ we get $i\left(g^{-\infty}\right)=a_{-}$.

Proposition 2.11 (Cannon-Thurston map unique). Let $G$ be a word-hyperbolic group acting as a non-elementary convergence group on a compact metrizable space $Z$. Then any two Cannon-Thurston maps $i, j: \partial G \rightarrow Z$, if they exist, must be equal.

Proof. Since $i, j$ are continuous, they are determined by what they do to a dense set of points. The set of attracting endpoints of any infinite order element $g \in G$ and its conjugates $\left\{g^{h}\right\}_{h \in G}$ forms such a dense set. By Lemma 2.10, $i$ and $j$ must agree on this set, hence must be equal.

In the situation where Proposition 2.11 applies, if a Cannon-Thurston map $i$ : $\partial G \rightarrow Z$ exists, we will refer to $i$ as the Cannon-Thurston map. 
There is a particular geometric situation where the Cannon-Thurston map has a more natural geometric meaning:

Proposition 2.12. Let $G$ be a non-elementary word-hyperbolic group equipped with a properly discontinuous (but not necessarily cocompact) isometric action of a proper Gromov-hyperbolic geodesic metric space $Y$, so that every element of infinite order acts as a loxodromic isometry of $Y$. Then the following hold:

(1) $\partial Y$ is compact and $G$ acts on $\partial Y$ as a convergence group without accidental parabolics. (Thus Proposition 2.11 applies.)

(2) Suppose the Cannon-Thurston map $i: \partial G \rightarrow \partial Y$ exists. Then for every $p \in Y$ the map

$$
f: G \cup \partial G \rightarrow Y \cup \partial Y
$$

given by $f(g)=$ gp for $g \in G$, and $f(x)=i(x)$ for $x \in \partial G$, is continuous for the hyperbolic compactification topologies on $G \cup \partial G$ and $Y \cup \partial Y$.

Proof. Part (1) is well known and due to Tukia 85.

For part (2), note that the topology on $G$ is discrete. Thus we only need to check continuity of $f$ at points of $\partial G$. Since $i$ is assumed to be continuous, it suffices to establish the following:

Claim. If $x \in \partial G$ and $\left(g_{n}\right)_{n \geq 1} \subset G$ is an infinite sequence of distinct elements of $G$ such that $\lim _{n \rightarrow \infty} g_{n}=x$ in $G \cup \partial G$, then $\lim _{n \rightarrow \infty} g_{n} p=i(x)$ in $Y \cup \partial Y$ for every $p \in Y$.

Assume that $x$ and $\left(g_{n}\right)$ are as in the Claim, but that the sequence $g_{n} p$ does not converge to $i(x)$ in $Y \cup \partial Y$. Since $G$ acts properly discontinuously on $Y$, it follows that, after replacing $g_{n}$ by a subsequence, we have $\lim _{n \rightarrow \infty} g_{n} p=z$ for some $z \in \partial Y$ such that $z \neq i(x)$. Then there exist a subsequence $g_{n_{k}}$ and points $a, b \in \partial G$ and $c, d \in \partial Y$ such that $\left(\left.g_{n_{k}}\right|_{\partial G \backslash\{a\}}\right)$ converges uniformly on compact sets to the constant map to $b$, and $\left.g_{n_{k}}\right|_{\partial Y \backslash\{c\}}$ converges uniformly on compact sets to the constant map to $d$. Moreover, the fact that $\lim _{n \rightarrow \infty} g_{n}=x$ implies that $x=b$ and, similarly, the fact that $\lim _{n \rightarrow \infty} g_{n} p=z$ implies that $z=d$ (see [85]). Since the set $i(\partial G)$ is infinite, we can find $y \in \partial G$ such that $y \neq a$ and $i(y) \neq c$. Then, on one hand, we have $\lim _{k \rightarrow \infty} g_{n_{k}} i(y)=d=z$. On the other hand, $\lim _{k \rightarrow \infty} g_{n_{k}} y=b=x$ and therefore, by continuity of $i$, we have $\lim _{k \rightarrow \infty} g_{n_{k}} i(y)=i(x)$. This contradicts $z \neq i(x)$.

A general result of $\mathrm{Mj}$ shows that in this situation injectivity of the CannonThurston map is equivalent to the orbit map $X \rightarrow Y$ being a quasi-isometric embedding [76, Lemma 2.5]:

Proposition 2.13. Let $G$ and $Y$ be as in Proposition 2.12 and let $p \in Y$. Then the following conditions are equivalent:

(1) The Cannon-Thurston map $i: \partial G \rightarrow \partial Y$ exists and is injective.

(2) The orbit map $G \rightarrow Y, g \mapsto g p$, is a quasi-isometric embedding.

Proof. As noted above, this proposition holds by [76, Lemma 2.5]. The proposition also follows directly from the older result of Swenson [83. Indeed, (2) obviously implies (1). Thus assume that (1) holds and that the Cannon-Thurston map $i$ : $\partial G \rightarrow \partial Y$ exists and is injective. Since both $\partial G$ and $\partial Y$ are compact and Hausdorff, 
the map $i$ is a $G$-equivariant homeomorphism between $\partial G$ and $Z^{\prime}=i(\partial G)$. Since $G$ is word-hyperbolic, every point of $\partial G$ is conical. Therefore every point of $Z^{\prime}$ is conical for the $G$-action on $Z^{\prime}$ and hence, by Lemma 2.6, also for the action of $G$ on $\partial Y$. The main result of Swenson 83 then implies that the orbit map $G \rightarrow Y$, $g \mapsto g p$, is a quasi-isometric embedding.

Proposition 2.13 implies, in particular, that if $G_{1}$ is a word-hyperbolic subgroup of a word-hyperbolic group $G_{2}$ and if the Cannon-Thurston map $i: \partial G_{1} \rightarrow \partial G_{2}$ exists and is injective, then $G_{1}$ is quasiconvex in $G_{2}$.

\section{Algebraic laminations}

If $G$ is a word-hyperbolic group, we denote $\partial^{2} G:=\{(z, s) \in \partial G \times \partial G \mid z \neq s\}$. The set $\partial^{2} G$ is equipped with the subspace topology from the product topology on $\partial G \times \partial G$. The group $G$ has a natural diagonal action on $\partial^{2} G$ : for $g \in G$ and $(z, s) \in \partial^{2} G$ we have $g(z, s):=(g z, g s)$. Let $\partial G \times \partial G \rightarrow \partial G \times \partial G$ be the "flip" map given by $j:(x, y) \mapsto(y, x)$ for $(x, y) \in \partial G$.

Definition 3.1 (Algebraic lamination). Let $G$ be a word-hyperbolic group. An algebraic lamination on $G$ is a subset $L \subseteq \partial^{2} G$ such that $L$ is closed in $\partial^{2} G$, flipinvariant and $G$-invariant. A pair $(x, y) \in L$ is called a leaf of $L$. An element $x \in \partial G$ is called an end of $L$ if there exists $y \in \partial G, y \neq x$, such that $(x, y) \in L$.

For an algebraic lamination $L$ on $G$ denote by $\operatorname{End}(L)$ the set of all ends of $L$. Note that $\operatorname{End}(L)$ is a $G$-invariant subset of $\partial G$.

Definition 3.2 (Lamination and relation associated to a Cannon-Thurston map). Let $G$ be a word-hyperbolic group and let $Z$ be a compact metrizable space equipped with a convergence action of $G$ such that the Cannon-Thurston map $i: \partial G \rightarrow Z$ exists. Denote

$$
L_{i}:=\{(x, y) \in \partial G \times \partial G \mid i(x)=i(y), x \neq y\} .
$$

Since $i$ is continuous and $G$-equivariant, $L_{i}$ is a closed $G$-invariant and flip-invariant subset of $\partial^{2} G$. Thus $L_{i}$ is an algebraic lamination on $G$.

\section{Dynamical Characterization}

Suppose $G$ is a word-hyperbolic group acting as a non-elementary convergence group on $Z$, and let $i: \partial G \rightarrow Z$ be a Cannon-Thurston map. We say that a point $x \in \partial G$ is asymptotic to $L_{i}$ if for every conical sequence $\left\{g_{n}\right\}_{n=1}^{\infty}$ for $x$ with pole pair $\left(x_{-}, x_{+}\right)$we have $\left(x_{-}, x_{+}\right) \in L_{i}$, that is, $i\left(x_{-}\right)=i\left(x_{+}\right)$.

In this section, we prove the first theorem from the introduction.

Theorem $\mathbf{A}$, Suppose $G$ is word-hyperbolic and acts on the compact, metrizable space $Z$ as a non-elementary convergence group, and suppose $i: \partial G \rightarrow Z$ is a Cannon-Thurston map. Let $z \in i(\partial G)$. Then:

(1) The point $z \in Z$ is not a conical limit point for the action of $G$ on $Z$ if and only if some point $x \in i^{-1}(z)$ is asymptotic to $L_{i}$.

(2) If $\left|i^{-1}(z)\right|>1$, then any $x \in i^{-1}(z)$ is asymptotic to $L_{i}$, and hence $z$ is non-conical.

In the rest of this section, we assume the hypotheses of the theorem. Our first lemma shows that, up to subsequences, conical sequences in $G$ for $Z$ must come from conical sequences for $\partial G$. 
Lemma 4.1. Suppose that $z \in Z$ is a conical limit point and that $\left(g_{n}\right)_{n \geq 1}$ is a conical sequence for $z$. Then there exist $x \in i^{-1}(z)$ and a subsequence $\left(g_{n_{k}}\right)_{k \geq 1}$ which is a conical sequence for $x$. Moreover, if $\left(x_{-}, x_{+}\right)$is the pole pair for $\left(g_{n_{k}}\right)$ and $x$, then $\left(i\left(x_{-}\right), i\left(x_{+}\right)\right)$is the pole pair for $\left(g_{n_{k}}\right)$ and $z$, and, in particular, $i\left(x_{-}\right) \neq i\left(x_{+}\right)$.

Proof. Without loss of generality, we may assume that the action of $G$ on $Z$ is minimal, so $i(\partial G)=Z$. Let $\left(z_{-}, z_{+}\right)$be the pole pair for $z$ and $\left(g_{n}\right)$. This is also a pole pair for any subsequence of $\left(g_{n}\right)$.

By the convergence property, there exist subsequence data $\left(x, x_{-}, g_{n_{k}}\right)$ such that $\left(\left.g_{n_{k}}\right|_{\partial G \backslash\{x\}}\right)_{k \geq 1}$ converges locally uniformly to the constant map to $x_{-}$. By passing to a further subsequence, we may assume that $\lim _{k \rightarrow \infty} g_{n_{k}}(x)=x_{+}$, for some $x_{+} \in \partial G$ (possibly equal to $x_{-}$).

Since $i$ is continuous, it follows that for any $y \in \partial G$, we have

$$
i\left(\lim _{k \rightarrow \infty} g_{n_{k}}(y)\right)=\lim _{k \rightarrow \infty} g_{n_{k}}(i(y)) .
$$

From this, we see that if $y \in \partial G \backslash\left(i^{-1}(z) \cup\{x\}\right)$, then $i\left(x_{-}\right)=i\left(\lim _{k \rightarrow \infty} g_{n_{k}}(y)\right)=z_{-}$. Furthermore, since any $y \in \partial G \backslash\{x\}$ has $\lim _{k \rightarrow \infty} g_{n_{k}}(i(y))=i\left(x_{-}\right)=z_{-}$, it follows that $i(\partial G \backslash\{x\}) \subset Z \backslash\{z\}$; that is, $i(x)=z$. Finally, we have

$$
i\left(x_{+}\right)=i\left(\lim _{k \rightarrow \infty} g_{n_{k}}(x)\right)=\lim _{k \rightarrow \infty} g_{n_{k}}(z)=z_{+} .
$$

Therefore $i\left(x_{+}\right)=z_{+} \neq z_{-}=i\left(x_{-}\right)$, and so $x_{+} \neq x_{-}$and $\left\{g_{n_{k}}\right\}$ is a conical sequence for $x$ with pole pair $\left(x_{-}, x_{+}\right)$.

Proof of Theorem A, To prove part (1), first suppose that $z \in Z$ is conical. Let $\left(g_{n}\right)_{n \geq 1}$ be a conical sequence for $z$ with pole pair $\left(z_{-}, z_{+}\right)$. According to Lemma 4.1. there exist $x \in i^{-1}(z)$ and a subsequence $\left(g_{n_{k}}\right)_{k \geq 1}$ which is a conical sequence for $x$ with pole pair $\left(x_{-}, x_{+}\right)$. Since $i\left(x_{-}\right)=z_{-} \neq z_{+}=i\left(x_{+}\right)$, it follows that $x$ is not asymptotic to $L_{i}$.

Now suppose $x$ is not asymptotic to $L_{i}$ and let $\left\{g_{n}\right\}_{n=1}^{\infty}$ be any conical sequence for $x$ with pole pair $\left(x_{-}, x_{+}\right)$such that $i\left(x_{-}\right) \neq i\left(x_{+}\right)$. Because the action of $G$ on $Z$ is a convergence action, there exist a subsequence $\left(g_{n_{k}}\right)_{k \geq 1}$ and $z, z_{-} \in Z$ such that on $Z \backslash\{z\}, g_{n_{k}}$ converges locally uniformly to $z_{-}$. For any $y \in \partial G \backslash\{x\}$ we have $\lim _{k \rightarrow \infty} g_{n_{k}}(i(y))=i\left(x_{-}\right)$. Thus taking $y \notin i^{-1}(z)$, this implies $i\left(x_{-}\right)=z_{-}$. On the other hand, $\lim _{k \rightarrow \infty} i\left(g_{n_{k}}(x)\right)=i\left(x_{+}\right) \neq i\left(x_{-}\right)=z_{-}$by assumption. It follows that $i(x)=z$ (since anything else must converge to $z_{-}$on applying $g_{n_{k}}$ ). Therefore, setting $z_{+}=i\left(x_{+}\right)$, it follows that $\left\{g_{n_{k}}\right\}$ is a conical sequence for $z$ with pole pair $\left(z_{-}, z_{+}\right)$, and $z$ is a conical limit point. Thus part (1) of Theorem $\mathrm{A}$ is proved.

For part (2) of Theorem $\mathrm{A}$ we suppose $\left|i^{-1}(z)\right|>1$, and prove that any $x \in i^{-1}(z)$ is asymptotic to $L_{i}$. For this, let $y \in i^{-1}(z)$ be any other point with $y \neq x$. Let $\left(g_{n}\right)_{n \geq 1}$ be a conical sequence for $x$ with pole pair $\left(x_{-}, x_{+}\right)$. Then $\lim _{n \rightarrow \infty} g_{n}(x)=x_{+}$ and $\lim _{n \rightarrow \infty} g_{n}(y)=x_{-}$. Since $L_{i}$ is $G$-invariant, $i\left(g_{n}(x)\right)=i\left(g_{n}(y)\right)$ and since $L_{i}$ is closed (or equivalently, the algebraic lamination $L_{i}$ is closed), it follows that $i\left(x_{-}\right)=i\left(x_{+}\right)$. Since $\left(g_{n}\right)$ was an arbitrary conical sequence for $x$, the point $x$ is asymptotic to $L_{i}$, as required. Hence, by part (1), $z$ is not a conical limit point for the action of $G$ on $Z$. 


\section{Geometric Characterization}

Definition 5.1 (Coarse leaf segments). Let $G$ be a word-hyperbolic group and let $L \subseteq \partial^{2} G$ be an algebraic lamination on $G$.

Let $X$ be a $\delta$-hyperbolic (where $\delta \geq 1$ ) proper geodesic metric space equipped with a properly discontinuous cocompact isometric action of $G$, so that $\partial G$ is naturally identified with $\partial X$.

For an algebraic lamination $L$ on $G$, a geodesic segment $\tau=[a, b]$ in $X$ is called a coarse $X$-leaf segment of $L$ if there exist a pair $(x, y) \in L$ and a bi-infinite geodesic $\gamma$ from $x$ to $y$ in $X$ such that $\tau$ is contained in the $2 \delta$-neighborhood of $\gamma$.

If $C \geq 0$, for a geodesic segment $\tau=[a, b]$ of length $\geq 2 C$, the $C$-truncation of $\tau$ is defined as $\left[a^{\prime}, b^{\prime}\right] \subseteq[a, b]$ where $a^{\prime}, b^{\prime} \in[a, b]$ are such that $d\left(a, a^{\prime}\right)=d\left(b, b^{\prime}\right)=C$.

Theorem B. Let $G$ be a word-hyperbolic group and let $Z$ be a compact metrizable space equipped with a non-elementary convergence action of $G$ such that the Cannon-Thurston map $i: \partial G \rightarrow Z$ exists and such that $i$ is not injective. Let $X$ be a $\delta$-hyperbolic (where $\delta \geq 1$ ) proper geodesic metric space equipped with a properly discontinuous cocompact isometric action of $G$ (so that $\partial G$ is naturally identified with $\partial X)$.

Let $x \in \partial G$, let $z=i(x) \in Z$ and let $\rho$ be a geodesic ray in $X$ limiting to $x$.

Then the following are equivalent:

(1) The point $z$ is a conical limit point for the action of $G$ on $Z$.

(2) There exist a geodesic segment $\tau=[a, b]$ in $X$ of length $\geq 100 \delta$ and an infinite sequence of distinct elements $g_{n} \in G$ such that the $20 \delta$-truncation $\tau^{\prime}$ of $\tau$ is not a coarse $X$-leaf segment of $L_{i}$ and such that for each $n \geq 1$ the segment $g_{n} \tau$ is contained in a $6 \delta$-neighborhood of $\rho$. [Note that this condition automatically implies that $\lim _{n \rightarrow \infty} g_{n} a=\lim _{n \rightarrow \infty} g_{n} b=x$.]

Proof. Suppose first that (1) holds and that $z$ is a conical limit point for the action of $G$ on $Z$. Since by assumption $i$ is not injective, there exists a pair $\left(y^{\prime}, y\right) \in L_{i}$ such that $i(y)=i\left(y^{\prime}\right)$. Denote $s=i(y)=i\left(y^{\prime}\right)$. By translating by an element of $g$ if necessary, we may also assume that $s \neq z$.

Since $i(x)=z$ and $z \neq s$, we have $x \neq y$. Note that $y \in \operatorname{End}\left(L_{i}\right)$.

Consider a geodesic $\gamma$ from $y$ to $x$ in $X$. Since $z$ is conical, by Proposition 2.7 there exists an infinite sequence of distinct elements $h_{n} \in G$ such that $\lim _{n \rightarrow \infty} h_{n}(s, z)=\left(s_{\infty}, z_{\infty}\right)$ for some $s_{\infty}, z_{\infty} \in Z$ such that $s_{\infty} \neq z_{\infty}$. After passing to a further subsequence, we may assume that $\lim _{n \rightarrow \infty} h_{n} x=x_{\infty}$ and $\lim _{n \rightarrow \infty} h_{n} y=y_{\infty}$ for some $x_{\infty}, y_{\infty} \in \partial G=\partial X$. By continuity of $i$ we have $i\left(x_{\infty}\right)=z_{\infty}$ and $i\left(y_{\infty}\right)=s_{\infty}$. In particular, this means that $x_{\infty} \neq y_{\infty}$ and that $\lim _{n \rightarrow \infty} h_{n}(y, x)=\left(y_{\infty}, x_{\infty}\right)$ in $\partial^{2} G$. Let $\gamma_{\infty}$ be a geodesic in $X$ from $y_{\infty}$ to $x_{\infty}$.

Then there exists a sequence of finite subsegments $\tau_{n}=\left[q_{n}, r_{n}\right]$ of $\gamma$ and a sequence of subsegments $\left[a_{n}, b_{n}\right]$ of $\gamma_{\infty}$ with the following properties:

a) We have $\lim _{n \rightarrow \infty} a_{n}=y_{\infty}, \lim _{n \rightarrow \infty} b_{n}=x_{\infty}$ and $\left[a_{n}, b_{n}\right]$ is a subsegment of $\left[a_{n+1}, b_{n+1}\right]$.

b) We have either $\lim _{n \rightarrow \infty} q_{n}=\lim _{n \rightarrow \infty} r_{n}=x$ or $\lim _{n \rightarrow \infty} q_{n}=\lim _{n \rightarrow \infty} r_{n}=$ $y$.

c) For all $n \geq 1$ the paths $h_{n}\left[q_{n}, r_{n}\right]$ and $\left[a_{n}, b_{n}\right]$ are $4 \delta$-close.

d) We have $h_{n} q_{n} \rightarrow_{n \rightarrow \infty} y_{\infty}$ and $h_{n} r_{n} \rightarrow_{n \rightarrow \infty} x_{\infty}$. 
If $\lim _{n \rightarrow \infty} q_{n}=\lim _{n \rightarrow \infty} r_{n}=y$, then, since $y \in \operatorname{End}\left(L_{i}\right)$ and since $L_{i} \subseteq \partial^{2} G$ is closed, it follows that $\left(y_{\infty}, x_{\infty}\right) \in L_{i}$. Therefore $z_{\infty}=i\left(x_{\infty}\right)=i\left(y_{\infty}\right)=s_{\infty}$, which contradicts the fact that $s_{\infty} \neq p_{\infty}$. Therefore $\lim _{n \rightarrow \infty} q_{n}=\lim _{n \rightarrow \infty} r_{n}=x$. Since $s_{\infty} \neq z_{\infty}$, it follows that $\left(y_{\infty}, x_{\infty}\right) \notin L_{i}$. Then there exists $m \geq 1$ such that $d\left(a_{m}, b_{m}\right) \geq 100 \delta$ and such that for $\tau:=\left[a_{m}, b_{m}\right]$ the $20 \delta$-truncation $\tau^{\prime}=$ $\left[a_{m}^{\prime}, b_{m}^{\prime}\right] \subseteq \gamma_{\infty}$ of $\tau$ is not a coarse $X$-leaf segment of $L_{i}$. By construction, for every $n \geq m, h_{n}^{-1} \tau$ is contained in a $4 \delta$-neighborhood of $\left[q_{n}, r_{n}\right]$ and hence, for all sufficiently large $n$, in a $6 \delta$-neighborhood of $\rho$. Thus we have verified that (1) implies (2).

Suppose now that (2) holds and that there exist a geodesic segment $\tau=[a, b]$ in $X$ of length $\geq 100 \delta$ and an infinite sequence of distinct elements $g_{n} \in G$ such that the $10 \delta$-truncation $\tau^{\prime}=\left[a^{\prime}, b^{\prime}\right]$ of $\tau$ is not an $X$-leaf segment of $L_{i}$ and such that for each $n \geq 1$ the segment $g_{n} \tau$ is contained in a $6 \delta$-neighborhood of $\rho$. We claim that $z$ is a conical limit point for the action of $G$ on $Z$. In view of Lemma 2.6, we may assume that $i(\partial G)=Z$.

Indeed, let $s \in Z$ be arbitrary such that $s \neq z$. Recall that $i(x)=z$. Choose $y \in \partial G$ such that $i(y)=s$. Thus $x \neq y$. Consider the bi-infinite geodesic $\gamma$ from $y$ to $x$ in $X$. Recall that $\rho$ is a geodesic ray in $X$ limiting to $x$.

After chopping-off a finite initial segment of $\rho$ if necessary, we may assume that there is a point $w \in \gamma$ such that the ray $\rho^{\prime}$ from $w$ to $x$ contained in $\gamma$ is $2 \delta$-close to $\rho$. By assumption, for every $n \geq 1$ the geodesic $g_{n}^{-1} \gamma$ from $g_{n}^{-1} y$ to $g_{n}^{-1} x$ contains a subsegment which is $8 \delta$-close to $\tau$. By compactness, after passing to a further subsequence, we may assume that $\lim _{n \rightarrow \infty} g_{n}^{-1} y=y_{\infty}$ and $\lim _{n \rightarrow \infty} g_{n}^{-1} x=x_{\infty}$ for some distinct points $x_{\infty}, y_{\infty} \in \partial G$. Let $\gamma_{\infty}$ be a geodesic from $y_{\infty}$ to $x_{\infty}$ in $X$.

We have $\tau^{\prime}=\left[a^{\prime}, b^{\prime}\right] \subseteq[a, b]=\tau$ with $d\left(a, a^{\prime}\right)=d\left(b, b^{\prime}\right)=20 \delta$. Since $\tau$ is contained in the $8 \delta$-neighborhood of $g_{n}^{-1} \gamma$, the segment $\tau^{\prime}$ is contained in a $2 \delta$ neighborhood of $\gamma_{\infty}$, and $\tau^{\prime}$ has length $\geq 50 \delta$. Since by assumption $\tau^{\prime}$ is not a coarse $X$-leaf segment of $L_{i}$, it follows that $\left(y_{\infty}, x_{\infty}\right) \notin L_{i}$ and hence $i\left(x_{\infty}\right) \neq$ $i\left(y_{\infty}\right)$. Denote $z_{\infty}=i\left(x_{\infty}\right)$ and $s_{\infty}=i\left(y_{\infty}\right)$. Since $i(x)=z, i(y)=s$ and since $\lim _{n \rightarrow \infty} g_{n}^{-1} y=y_{\infty}$ and $\lim _{n \rightarrow \infty} g_{n}^{-1} x=x_{\infty}$, the continuity of $i$ implies that $\lim _{n \rightarrow \infty} g_{n}^{-1}(s, z)=\left(s_{\infty}, z_{\infty}\right)$. Since $s_{\infty} \neq z_{\infty}$, Proposition 2.7 implies that $z$ is indeed a conical limit point for the action of $G$ on $Z$, as required.

\section{INJECTIVE, NON-CONICAL LIMIT POINTS}

Here we prove that injective non-conical limit points occur quite often.

Theorem C. Suppose a word-hyperbolic group $G$ acts on a compact metrizable space $Z$ as a non-elementary convergence group without accidental parabolics, and suppose that there exists a non-injective Cannon-Thurston map $i: \partial G \rightarrow Z$. Then there exists a non-conical limit point $z \in Z$ with $\left|i^{-1}(z)\right|=1$.

Suppose that $G$ is a hyperbolic group acting as a non-elementary convergence group on $Z$ as in the statement of the theorem, from which it follows that $G$ is also non-elementary. Fix a finite generating set $\mathcal{S}$ for $G$, such that $\mathcal{S}=\mathcal{S}^{-1}$, and let $X$ be the Cayley graph of $G$ with respect to $\mathcal{S}$, endowed with the usual geodesic metric in which every edge has length 1 . Then $X$ is $\delta$-hyperbolic for some $\delta>0$. We denote the length of a geodesic segment $\sigma$ in $X$ as $|\sigma|$. Recall that for $r>0$ 
an $r$-local geodesic in $X$ is a path $\alpha$ parameterized by arclength such that every subsegment of $\alpha$ of length $r$ is a geodesic. There exist integers $r>0$ and $D>0$ such that any $r$-local geodesic in $X$ is quasigeodesic (with constants depending only on $r$ and $\delta$ ), and such that the Hausdorff distance between an $r$-local geodesic and the geodesic with the same endpoints is at most $D$; see e.g. 21, Part III, Chapter 1].

Given an algebraic lamination $L \subset \partial^{2} G$, define the geodesic realization of $L$ with respect to $\mathcal{S}$, denoted $\mathcal{L}$, as the set of all $\ell \subset X$ such that there exist $x, y \in \partial G$ with $(x, y) \in L$ such that $\ell$ is a bi-infinite geodesic in $X$ from $x$ to $y$.

Convention 6.1. For the remainder of this section, we assume $G, Z, i$ are as in the statement of the theorem, $\mathcal{S}, X, \delta, r, D$ are as above, $L_{i}$ is the algebraic lamination associated to $i$ as in Definition 3.2 and $\mathcal{L}_{i}$ denotes the geodesic realization of $L_{i}$.

Given integers $p \geq 1$, a $p$-periodic, $r$-local geodesic in $X$ is a bi-infinite $r$-local geodesic $\gamma$ in $X$ for which some element $g \in G$ acts on $\gamma$ translating a distance $p$ along $\gamma$. As $\gamma$ is a quasigeodesic, it follows that $g$ has infinite order (and $\gamma$ is a quasigeodesic axis for $g$ in $X$ ).

We will use the following lemma in the proof of the theorem.

Lemma 6.2. For any $p \geq 1$, there exists $c(p) \geq 1$ with the following property. If $\gamma$ is a p-periodic, $r$-local geodesic in $X$ and $\ell \in \mathcal{L}_{i}$ contains a segment $\sigma \subset \gamma$ in its $(\delta+D)$-neighborhood, then $|\sigma|<c(p)$.

Proof. It suffices to prove this statement for any fixed $p$-periodic, $r$-local geodesic $\gamma$ in $X$ (since, for a given $p$, there are only finitely many $G$-orbits of such $\gamma$ ). Translating such $\gamma$ if necessary, we may assume that $\gamma$ passes through the identity 1 in $G \subset X$. Let $g \in G$ be a translation of length $p$ along $\gamma$.

Now if the requisite $c(p)$ does not exist, then there exists a sequence $\left\{\ell_{n}\right\}_{n \geq 1}$ of elements of $\mathcal{L}_{i}$ so that each $\ell_{n}$ contains a segment $\sigma_{n} \subseteq \gamma$ of length at least $n$ in its $(\delta+D)$-neighborhood. Since $\mathcal{L}_{i}$ is $G$-invariant, after applying an appropriate power of $g$ to $\ell_{n}$ if necessary, we can assume that the midpoint of $\sigma_{n}$ lies within distance $p$ of $1 \in G$. In particular, for $n>p, 1 \in \sigma_{n}$ and $\ell_{n}$ is within $\delta+D$ of 1 . Passing to a subsequence, we can assume that $\ell_{n} \rightarrow \ell \in \mathcal{L}_{i}$ as $n \rightarrow \infty$ (since $L_{i}$ is closed). On the other hand, since $\sigma_{n} \rightarrow \gamma$, as $n \rightarrow \infty$, we see that $\gamma$ is within $\delta+D$ of $\ell$. Therefore, $\ell$ and $\gamma$ have the same endpoints on $\partial G$. Since the endpoints of $\gamma$ are the fixed points of $g$, and the endpoints of $\ell$ are identified by $i$, it follows that $g$ is an accidental parabolic for the action on $Z$, yielding a contradiction.

We are now ready for the proof of the theorem.

Proof of Theorem C. Let $\ell \in \mathcal{L}_{i}$ be a bi-infinite geodesic in $\mathcal{L}_{i}$ and let $\ell_{+} \subset \ell$ be a geodesic ray contained in $\ell$. We can view $\ell_{+}$as a semi-infinite word over the alphabet $\mathcal{S}$.

For any $m \geq r$, let $v_{m} \in \mathcal{S}^{*}$ be a word of length $m$ which occurs (positively) infinitely often in $\ell_{+}$. Such a word exists, for every $m$, by the pigeonhole principle. Now we define several additional families of subwords of $\ell_{+}$. These subwords will serve as the building blocks for a new $r$-local geodesic (hence quasigeodesic) infinite ray. 
For each $m \geq r$ :

(1) Let $u_{m}$ be any subword of $\ell_{+}$of length at least $m$ so that $v_{m} u_{m} v_{m}$ occurs in $\ell_{+}$. Such $u_{m}$ exists because $v_{m}$ occurs in $\ell_{+}$infinitely often.

(2) Let $t_{m}$ be any non-empty word so that $v_{m} t_{m} v_{m+1}$ occurs in $\ell_{+}$. These exist for the same reason as $u_{m}$.

(3) Put $\alpha_{m}=v_{m} u_{m}$.

Let $p_{m}=\left|\alpha_{m}\right|$, and let $\kappa_{m}>0$ be an integer such that $\kappa_{m} p_{m}>c\left(p_{m}\right)$. Note that, since $v_{m} u_{m} v_{m}$ is a subword of a geodesic ray $\ell_{+}$with $\left|u_{m}\right|,\left|v_{m}\right| \geq m$, it follows that the word $\alpha_{m}=v_{m} u_{m}$ is cyclically reduced and that for every $k \geq 1$ every subword of length $m$ in $\alpha_{m}^{k}$ is a geodesic and occurs as a subword of $v_{m} u_{m} v_{m}$ and thus of $\ell_{+}$.

Now consider the following semi-infinite word (which we also view as a semiinfinite path in $X$ with origin $1 \in G$ ):

$$
w_{\infty}:=\alpha_{r}^{\kappa_{r}} v_{r} t_{r} \alpha_{r+1}^{\kappa_{r+1}} v_{r+1} t_{r+1} \alpha_{r+2}^{\kappa_{r+2}} \cdots .
$$

This word $w_{\infty}$ is naturally a union of subwords of the following forms:

(1) $\alpha_{m}$, which is a subword of $\ell_{+}$.

(2) $v_{m} t_{m} v_{m+1}$, which is a subword of $\ell_{+}$.

(3) $\alpha_{m}^{\kappa_{m}}$, which is a word of length $p_{m} \kappa_{m}>c\left(p_{m}\right)$, is contained in a $p_{m}$-periodic, $r$-local geodesic. As such, the word $\alpha_{m}^{\kappa_{m}}$ is not contained in a $(D+\delta)$ neighborhood of any $\ell^{\prime} \in \mathcal{L}_{i}$, by Lemma 6.2. However, any subword of length $m$ of $\alpha_{m}^{\kappa_{m}}$ occurs in $\ell_{+}$.

Moreover, any subword $v$ of $w_{\infty}$ of length $r$ is contained in at least one such word, and thus $v$ occurs as a subword of $\ell_{+}$. Therefore $w_{\infty}$ is an $r$-local geodesic in $X$ and hence a global quasigeodesic in $X$. Furthermore, note that as $m$ tends toward infinity, the lengths of the words $\alpha_{m}$ and $v_{m} t_{m} v_{m+1}$ tend to infinity. Denote the endpoint of $w_{\infty}$ in $\partial G$ by $x$.

First, we claim that $\left|i^{-1}(i(x))\right|=1$. If this were not the case, then the ray $w_{\infty}$ would be asymptotic to (i.e. have a finite Hausdorff distance to) an infinite ray $\ell_{+}^{\prime} \subset \ell^{\prime}$ for some geodesic $\ell^{\prime} \in \mathcal{L}_{i}$. In this case, a subray $w_{\infty}^{\prime} \subset w_{\infty}$ would be contained in the $(\delta+D)$-neighborhood of $\ell_{+}^{\prime}$. Since this ray contains arcs labeled $\alpha_{m}^{\kappa_{m}}$ for $m$ sufficiently large, this contradicts Lemma 6.2.

Second, we claim that $i(x)$ is non-conical. To prove this, let $\gamma$ be an $r$-local geodesic containing $w_{\infty}$ as a subray. For example, let $\gamma$ be the concatenation of the ray which is $\alpha_{r}^{-\infty}$ with $w_{\infty}$. One endpoint of $\gamma$ is $x$, and we denote the other by $y$. Let $\left(g_{n}\right)_{n \geq 1}$ be any convergence sequence for $x$ with pole pair $\left(x_{-}, x_{+}\right)$. Then $g_{n}(x) \rightarrow x_{+}$and $g_{n}(y) \rightarrow x_{-}$. Since $x_{-} \neq x_{+}$, after passing to a subsequence $\left(g_{n_{k}}\right)$, the $r$-local geodesics $g_{n_{k}} \gamma$ must converge to an $r$-local geodesic with endpoints $x_{-}, x_{+}$. After passing to a further subsequence (still denoted $\left(g_{n_{k}}\right)$ ), it follows that $g_{n_{k}} \gamma$ converges to an $r$-local geodesic with endpoints $x_{-}, x_{+}$. Since $\left(g_{n_{k}}\right)$ is a convergence sequence for $x$, if $k$ is sufficiently large, any closest point $h_{k} \in g_{n_{k}} \gamma$ to 1 must have $g_{n_{k}}^{-1}\left(h_{k}\right) \in w_{\infty}$ with distance to the initial point of $w_{\infty}$ tending toward infinity. Passing to yet a further subsequence if necessary, we can assume that the subword $w_{k} \subset w_{\infty}$ of length $2 k$ centered on $g_{n_{k}}^{-1} h_{k}$ is a subword of $\ell_{+} \subset \ell$. Thus for all $k$ there exists $\ell_{k} \in \mathcal{L}_{i}$ (a translate of $\ell$ ) so that the segment $w_{k}$ is contained in $\ell_{k}$.

Now observe that $g_{n_{k}}\left(w_{k}\right)$ is a segment of $g_{n_{k}}\left(\ell_{k}\right) \in \mathcal{L}_{i}$. Because $g_{n_{k}}\left(w_{k}\right)$ is a geodesic of length $2 k$ centered on $h_{k}$, it follows that $g_{n_{k}}\left(w_{k}\right)$, and hence $g_{n_{k}}\left(\ell_{k}\right)$, 
converges to a geodesic with endpoints $\left(x_{-}, x_{+}\right)$as $k \rightarrow \infty$. However, $g_{n_{k}}\left(\ell_{k}\right)$ must converge to a leaf of $\mathcal{L}_{i}$ since $L_{i}$ is closed. Since $\left(g_{n}\right)$ was an arbitrary convergence sequence for $x, x$ is asymptotic to $L_{i}$, and by Theorem $\mathrm{A}$, $i(x)$ is non-conical.

\section{Controlled concentration points}

Definition 7.1. Let $G$ be a non-elementary torsion-free discrete subgroup of hyperbolic isometries acting on $\mathbb{H}^{n}$ and let $S_{\infty}^{n-1}$ be the ideal boundary of $\mathbb{H}^{n}$. A neighborhood $U \subset S_{\infty}^{n-1}$ of $x \in \Lambda(G)$ is called concentrated at $x$ if for every neighborhood $V$ of $x$, there exists an element $g \in G$ such that $x \in g(U)$ and $g(U) \subset V$. If such $g$ can always be chosen so that $x \in g(V)$, then we say $U$ is concentrated with control. A limit point $x$ in $\Lambda(G)$ is called a controlled concentration point if it has a neighborhood which is concentrated with control.

A geodesic ray in $\mathbb{H}^{n}$ is called recurrent with respect to $G$ if its image $\alpha$ in $M=\mathbb{H}^{n} / G$ by the covering projection is recurrent. Recall that a geodesic ray $\alpha$ parameterized by $[0, \infty)$ in $M$ is called recurrent if for any tangent vector $v=$ $\alpha^{\prime}\left(t_{0}\right), t_{0}>0$, in the unit tangent bundle $U T(M)$ of $M$, there exists an infinite sequence of times $\left\{t_{i}\right\}$ such that $\alpha^{\prime}\left(t_{i}\right)$ converges to $v$ in $U T(M)$. The main result of [3] is that controlled concentration points correspond to the endpoints of recurrent geodesic rays.

Theorem 7.2 (Aebischer, Hong and McCullough [3]). Let $G$ be as in Definition 7.1. Then for a limit point $x \in \Lambda(G)$, the following are equivalent:

(1) There is a recurrent geodesic ray whose endpoint is $x$.

(2) $x$ is a controlled concentration point.

(3) There exists a sequence $\left\{g_{n}\right\}$ of distinct elements of $G$ such that for any geodesic ray $\beta$ whose endpoint $\beta(\infty)$ is $x, g_{n}(\beta)$ converges to some geodesic ray whose endpoint is again $x$ up to taking a subsequence.

(4) There exists a sequence $\left\{g_{n}\right\}$ of distinct elements of $G$ and $y \in S_{\infty}^{n-1}$ with $y \neq x$ such that $g_{n} x \rightarrow x$ and $\left.g_{n}\right|_{S_{\infty}^{n} \backslash\{x\}}$ converges uniformly on compact subsets to the constant map to $y$.

From the last characterization of a controlled concentration point, it is clear that every controlled concentration point is conical, but the converse is not true in general. In fact [3. Prop. 5.1] gives an example of a conical limit point which is not a controlled concentration point in the case of a rank-2 Schottky group. It is also known that the set of controlled concentration points has full Patterson-Sullivan measure in $\Lambda(G)$ if $G$ is of divergence type. Note that for a geodesic lamination $\lambda$ in a hyperbolic surface $S$, a leaf of $\lambda$ is always a recurrent geodesic, and hence its endpoints are controlled concentration points.

The following proposition follows easily from condition (1) of Theorem 7.2

Proposition 7.3. A limit point $x$ in $\Lambda(G) \subset S_{\infty}^{n-1}$ is a controlled concentration point if there exists a geodesic ray $\beta$ in $\mathbb{H}^{n}$ which limits to $x$, and the $\omega$-limit set of $\beta$ in the geodesic foliation on the unit tangent bundle $U T(M)$ of $M=\mathbb{H}^{n} / G$ has only one minimal component.

We extend the notion of controlled concentration points to the case of hyperbolic groups.

Definition 7.4. Let $G$ be a non-elementary hyperbolic group. Then we say $x \in \partial G$ is a controlled concentration point if there exists a sequence $\left\{g_{n}\right\}$ of distinct elements 
of $G$ and $y \in \partial G$ with $y \neq x$ such that $g_{n} x \rightarrow x$ and $\left.g_{n}\right|_{\partial G \backslash\{x\}}$ converges locally uniformly to the constant map to $y$.

Proposition 7.5. Suppose $G$ is word-hyperbolic and acts on the compact, metrizable space $Z$ as a non-elementary convergence group, and suppose $i: \partial G \rightarrow Z$ is a Cannon-Thurston map. If a controlled concentrated point $x \in \partial G$ satisfies $\left|i^{-1}(i(x))\right|=1$, then $i(x) \in Z$ is conical.

Proof. Since $x$ is a controlled concentrated point, there exist $y \in \partial G$ with $y \neq x$ and a sequence $\left\{g_{n}\right\}$ of distinct elements in $G$ such that $\lim _{n \rightarrow \infty} g_{n} x=x$ and $\left(\left.g_{n}\right|_{\partial G \backslash\{x\}}\right)$ locally uniformly converges to the constant map to $y$. Note that $\left|i^{-1}(i(x))\right|=1$ implies $i(x) \neq i(y)$. Suppose $i(x) \in Z$ is not conical. Then by Proposition 2.7 there exists a subsequence $\left(g_{n_{k}}\right)$ of $\left(g_{n}\right)$ such that $\lim _{k \rightarrow \infty} g_{n_{k}} i(x)=\lim _{k \rightarrow \infty} g_{n_{k}} i(y)$ and hence by continuity

$$
(i(x), i(y))=\lim _{k \rightarrow \infty}\left(i\left(g_{n_{k}} x\right), i\left(g_{n_{k}} y\right)\right)=\lim _{k \rightarrow \infty}\left(g_{n_{k}} i(x), g_{n_{k}} i(y)\right)=(z, z)
$$

for some $z \in Z$, and hence $i(x)=i(y)$. This is a contradiction.

We can now prove the last theorem from the Introduction:

Theorem D, Let $G$ be a non-elementary torsion-free word-hyperbolic group. Then there exists $x \in \partial G$ which is not a controlled concentration point.

Proof. Kapovich [47] proved that, given a non-elementary torsion-free word-hyperbolic group $G$, there exists a word-hyperbolic group $G_{*}$ containing $G$ as a nonquasiconvex subgroup. Moreover, $G_{*}$ is constructed in [47] as an HNN-extension

$$
G_{*}=\left\langle G, t \mid t^{-1} K t=K_{1}\right\rangle
$$

where $K \leq G$ is a quasiconvex free subgroup of rank 2 and where $K_{1} \leq K$ is also free of rank 2 (and hence $K_{1}$ is also quasiconvex in $G$ ). Therefore, by a general result of Mitra 69] (see also [78]) about graphs of groups with hyperbolic edge and vertex groups, there does exist a Cannon-Thurston map $i: \partial G \rightarrow \partial G_{*}$. Since $G \leq G_{*}$ is not quasiconvex, Proposition 2.13 implies that the map $i$ is not injective. Therefore, by Theorem [C] there exists a non-conical limit point $z \in i(\partial G)$ with $\left|i^{-1}(z)\right|=1$. By Proposition $7.5, x=i^{-1}(z) \in \partial G$ is not a controlled concentration point.

\section{Appendix A. Descriptions of $L_{i}$}

There are several situations where the Cannon-Thurston map $i: \partial G \rightarrow Z$ is known to exist and where a more detailed description of the lamination $L_{i}$ is known. Theorem $\mathrm{A}$ and Theorem $\mathrm{B}$ may be useful in these contexts. The proof of Theorem 6.6 in 33 . uses Theorem B as a key ingredient in this way.

A.1. Kleinian representations of surface groups. Let $G$ be the fundamental group of a closed, orientable hyperbolic surface $S$. The universal covering of $S$ is isometric to the hyperbolic plane $\mathbb{H}^{2}$ with $G$ acting cocompactly by isometries, and so we can identify the Gromov boundary of $G$ with the circle at infinity $\partial G \cong S_{\infty}^{1}$. A faithful Kleinian representation $\rho: G \rightarrow \operatorname{PSL}(2, \mathbb{C}) \cong \operatorname{Isom}^{+}\left(\mathbb{H}^{3}\right)$ is an injective homomorphism with discrete image. This determines a convergence action of $G$ on $S_{\infty}^{2}$, and hence also on the limit set $\Lambda(G)$. The existence of a Cannon-Thurston map for such groups was first proved in the special case when $\rho(G)$ is the fiber subgroup 
of a hyperbolic 3-manifold fibering over the circle by Cannon and Thurston 25]. This was extended to include other classes of Kleinian representations of $G$ in 65,69 ] and then arbitrary faithful, Kleinian representations of $G$ in 77 .

The hyperbolic 3-manifold $M=\mathbb{H}^{3} / \rho(G)$ is homeomorphic to $S \times(-\infty, \infty)$ by the Tameness Theorem ([15], and [1,24] in more general settings) and thus $M$ has only two ends, $E_{+}$and $E_{-}$. Assume that $\rho(G)$ has no parabolics. Associated to each end is a (possibly empty) ending lamination $\lambda_{+}$and $\lambda_{-}$, which is a geodesic lamination on $S$, that is, a closed union of pairwise disjoint complete geodesics; see [27. for more on geodesic laminations and [23, 66, 87] for more on the ending laminations associated to ends of 3-manifolds. The pre-images $\widetilde{\lambda}_{ \pm} \subset \mathbb{H}^{2}$ of the ending laminations in $\mathbb{H}^{2}$ are geodesic laminations in $\mathbb{H}^{2}$, and the endpoints of the leaves determine a pair of algebraic laminations $L_{ \pm} \subset \partial^{2} G$. Set $\mathcal{R}_{+}, \mathcal{R}_{-} \subset \partial G \times \partial G$ to be the reflexive and transitive closures of the pair $L_{+}, L_{-}$, respectively. Then for the Cannon-Thurston map $i$ has $i(x)=i(y)$ if and only if $(x, y) \in \mathcal{R}_{+} \cup \mathcal{R}_{-}$ according to [25] in the original setting, 65. for the cases treated there, and in general in [71. Furthermore, the transitive closure adds only endpoints of finitely many $G$-orbits of leaves, and thus $L_{i}$ is equal to $L_{+} \cup L_{-}$, together with finitely many additional $G$-orbits of leaves (which correspond to the "diagonals" of the complementary components of $\widetilde{\lambda}_{+}$and $\left.\widetilde{\lambda}_{-}\right)$.

\section{A.2. Short exact sequences of hyperbolic groups. Let}

$$
1 \rightarrow G_{1} \rightarrow G_{2} \rightarrow Q
$$

be a short exact sequence of three word-hyperbolic groups, such that $G_{1}$ is nonelementary. In this case $G_{1}$ acts on $Z=\partial G_{2}$ as a non-elementary convergence group without accidental parabolics. Mitra 68 proved that in this case the CannonThurston map $i: \partial G_{1} \rightarrow \partial G_{2}$ does exist. Therefore the results of this paper, including Theorem B, do apply. In 67] Mitra also obtained a general geometric description of $L_{i}$ in this case in terms of the so-called "ending laminations".

We give here a brief description of the results of [67.

Given every $\xi \in \partial Q$, Mitra defines an "ending lamination" $\Lambda_{\xi} \subseteq \partial^{2} G_{1}$. To define $\Lambda_{\xi}$, Mitra starts with choosing a quasi-isometric section $r: Q \rightarrow G_{2}$ (he later proves that the specific choice of $r$ does not matter). Then given any $\xi \in \partial Q$, take a geodesic ray in $Q$ towards $\xi$ and let $\xi_{n}$ be the point at distance $n$ from the origin on that ray. Lift $\xi_{n}$ to $G_{2}$ by the section $r$ to get an element $g_{n}=r\left(\xi_{n}\right) \in G_{2}$. Conjugation by $g_{n}$ gives an automorphism $\varphi_{n}$ of $G_{1}$ defined as $\varphi_{n}(h)=g_{n} h g_{n}^{-1}$, $h \in G_{1}$. Now pick any non-torsion element $h \in G_{1}$. Then look at all $(x, y) \in \partial^{2} G_{1}$ such that there exists a sequence of integers $k_{n} \rightarrow \infty$ and of conjugates (with respect to conjugation in $\left.G_{1}\right) w_{n}$ of $\varphi_{k_{n}}(h)$ in $G_{1}$ such that $\lim _{n \rightarrow \infty}\left(w_{n}^{-\infty}, w_{n}^{\infty}\right)=(x, y)$ in $\partial^{2} G_{1}$. For a fixed non-torsion $h \in G_{1}$, the collection of all such $(x, y) \in \partial^{2} G_{1}$ is denoted $\Lambda_{\xi, h}$. Denote by $A$ the set of all elements of infinite order in $G_{1}$. Finally, put $\Lambda_{\xi}=\bigcup_{h \in A} \Lambda_{\xi, h}$. The main result of Mitra in [67] says that, in this case

$$
L_{i}=\bigcup_{\xi \in \partial Q} \Lambda_{\xi}
$$

For every $\xi \in \partial Q$ the subset $\Lambda_{\xi} \subseteq \partial^{2} G_{1}$ is an algebraic lamination on $G_{1}$, and Mitra refers to $\Lambda_{\xi}$ as the "ending lamination" on $G_{1}$ corresponding to $\xi$. Moreover, the 
arguments of Mitra actually imply that if $\xi_{1}, \xi_{2} \in \partial Q$ are distinct, then $\operatorname{End}\left(\Lambda_{\xi_{1}}\right) \cap$ $\operatorname{End}\left(\Lambda_{\xi_{2}}\right)=\varnothing$. Mitra also notes that for any $\xi \in \partial Q$ there exists a finite subset $B \subseteq A$ such that $\Lambda_{\xi}=\bigcup_{h \in B} \Lambda_{\xi, h}$.

In general, for a short exact sequence $(\ddagger)$ and $\xi \in \partial Q$, the "ending lamination" $\Lambda_{\xi} \subseteq \partial^{2} G_{1}$ can, at least a priori, be quite large and difficult to understand. This is the case even if $Q=\langle t\rangle \cong \mathbb{Z}$ is infinite cyclic, so that $\partial Q=\left\{t^{-\infty}, t^{\infty}\right\}$ consists of just two points. However, in some situations the laminations $\Lambda_{\xi}$ are well understood.

A.3. Hyperbolic extensions of free groups. In particular, let $N \geq 3$, let $\varphi \in \operatorname{Out}\left(F_{N}\right)$ be a fully irreducible atoroidal element and let $\Phi \in \operatorname{Aut}\left(F_{N}\right)$ be a representative of the outer automorphism class of $\varphi$ (see $[8,10,28,43,44,48,63$, for the relevant background). Then

$$
G=F_{N} \rtimes_{\Phi} \mathbb{Z}=\left\langle F_{N}, t \mid t w t^{-1}=\Phi(w), w \in F_{N}\right\rangle
$$

is word-hyperbolic and we have a short exact sequence $1 \rightarrow F_{N} \rightarrow G \rightarrow\langle t\rangle \rightarrow 1$. Thus, by 68, there does exist a Cannon-Thurston map $i: \partial F_{N} \rightarrow \partial G$. Using the results of Mitra 67] mentioned above as a starting point, Kapovich and Lustig proved in 53] that $\Lambda_{t^{\infty}}=\operatorname{diag}\left(L_{B F H}(\varphi)\right)=L\left(T_{-}\right)$and, similarly, $\Lambda_{t^{-\infty}}=$ $\operatorname{diag}\left(L_{B F H}\left(\varphi^{-1}\right)\right)=L\left(T_{+}\right)$. Here $L_{B F H}(\varphi) \subseteq \partial^{2} F_{N}$ is the "stable" lamination of $\varphi$, introduced by Bestvina, Feighn and Handel in [9], and $\operatorname{diag}\left(L_{B F H}\left(\varphi^{-1}\right)\right)$ is the "diagonal extension" of $L_{B F H}(\varphi)$, that is, the intersection of $\partial^{2} F_{N}$ with the equivalence relation on $\partial F_{N}$ generated by the relation $L_{B F H}(\varphi) \subseteq \partial^{2} F_{N}$ on $\partial F_{N}$. Also, here $L\left(T_{-}\right)$is the "dual algebraic lamination" (in the sense of [30, 32, 51]) corresponding to the "repelling" $\mathbb{R}$-tree $T_{-}$for $\varphi$ (the tree $T_{-}$is constructed using a train-track representative for $\varphi^{-1}$ and the projective class of $T_{-}$is the unique repelling fixed point for the right action of $\varphi$ on the compactified Outer space. Thus, in view of the discussion above, we have

$$
L_{i}=\operatorname{diag}\left(L_{B F H}(\varphi)\right) \cup \operatorname{diag}\left(L_{B F H}\left(\varphi^{-1}\right)\right)=L\left(T_{-}\right) \cup L\left(T_{+}\right)
$$

in this case. The stable lamination $L_{B F H}(\varphi)$ of $\varphi$ is defined quite explicitly in terms of a train-track representative $f: \Gamma \rightarrow \Gamma$ of $\varphi$. Thus a pair $(x, y) \in \partial^{2} F_{N}$ belongs to $L_{B F H}(\varphi)$ if and only if for every finite subpath $\tilde{v}$ of the geodesic from $x$ to $y$ in $\widetilde{\Gamma}$, the projection $v$ of $\tilde{v}$ to $\Gamma$ has the property that for some edge $e$ of $\Gamma$ and some $n \geq 1$ the path $v$ is a subpath of $f^{n}(e)$. Kapovich and Lustig also proved in 52 ] that $\operatorname{diag}\left(L_{B F H}(\varphi)\right)$ is obtained from $L_{B F H}(\varphi)$ by adding finitely many $F_{N}$ orbits of "diagonal" leaves $(x, y)$ of a special kind. These extra "diagonal leaves" play a similar role to the diagonals of ideal polygons given by complementary regions for the lift to $\mathbb{H}^{2}$ of the stable geodesic lamination of a pseudo-anosov homeomorphism of a closed hyperbolic surface.

In 33] Dowdall, Kapovich and Taylor generalize the above description of $L_{i}$ to the case of word-hyperbolic extensions $E_{\Gamma}$ of $F_{N}$ determined by purely atoroidal "convex cocompact" subgroups $\Gamma \leq \operatorname{Out}\left(F_{N}\right)$. See [33, Corollary 5.3] for details.

\section{ACKNOWLEDGEMEnTS}

The authors thank Victor Gerasimov and Leonid Potyagailo for their interest in the results of this paper and for bringing to their attention the paper [39] and explaining the relationship between the results of [39] and Theorem $\mathrm{A}$. The authors are grateful to Brian Bowditch for helpful discussions regarding conical limit points 
and the Cannon-Thurston map, and to Sergio Fenley, Darren Long, and Alan Reid for useful conversations about convergence actions and conical limit points. The authors thank the referee for useful comments.

\section{REFERENCES}

[1] I. Agol, Tameness of hyperbolic 3-manifolds, preprint, 2004; arXiv:0405568

[2] J. W. Anderson, P. Bonfert-Taylor, and E. C. Taylor, Convergence groups, Hausdorff dimension, and a theorem of Sullivan and Tukia, Geom. Dedicata 103 (2004), 51-67, DOI 10.1023/B:GEOM.0000013844.35478.e5. MR2034952

[3] B. Aebischer, S. Hong, and D. McCullough, Recurrent geodesics and controlled concentration points, Duke Math. J. 75 (1994), no. 3, 759-774, DOI 10.1215/S0012-7094-94-07523-6. MR.1291703

[4] O. Baker and T. R. Riley, Cannon-Thurston maps do not always exist, Forum Math. Sigma 1 (2013), e3, 11, DOI 10.1017/fms.2013.4. MR3143716

[5] O. Baker and T. Riley, Cannon-Thurston maps, subgroup distortion, and hyperbolic hydra, preprint, 2012; arXiv:1209.0815

[6] A. F. Beardon and B. Maskit, Limit points of Kleinian groups and finite sided fundamental polyhedra, Acta Math. 132 (1974), 1-12. MR0333164

[7] M. Bestvina and M. Feighn, A combination theorem for negatively curved groups, J. Differential Geom. 35 (1992), no. 1, 85-101. MR.1152226

[8] M. Bestvina and M. Handel, Train tracks and automorphisms of free groups, Ann. of Math. (2) 135 (1992), no. 1, 1-51, DOI 10.2307/2946562. MR1147956

[9] M. Bestvina, M. Feighn, and M. Handel, Laminations, trees, and irreducible automorphisms of free groups, Geom. Funct. Anal. 7 (1997), no. 2, 215-244, DOI 10.1007/PL00001618. MR1445386

[10] M. Bestvina, M. Feighn, and M. Handel, The Tits alternative for $\operatorname{Out}\left(F_{n}\right)$. I. Dynamics of exponentially-growing automorphisms, Ann. of Math. (2) 151 (2000), no. 2, 517-623, DOI 10.2307/121043. MR.1765705

[11] M. Bestvina and M. Feighn, A hyperbolic Out $\left(F_{n}\right)$-complex, Groups Geom. Dyn. 4 (2010), no. 1, 31-58, DOI 10.4171/GGD/74. MR2566300

[12] M. Bestvina and M. Feighn, Hyperbolicity of the complex of free factors, Adv. Math. 256 (2014), 104-155, DOI 10.1016/j.aim.2014.02.001. MR3177291

[13] M. Bestvina and P. Reynolds, The boundary of the complex of free factors, Duke Math. J. 164 (2015), no. 11, 2213-2251, DOI 10.1215/00127094-3129702. MR3385133

[14] O. Bogopolski, Introduction to group theory, EMS Textbooks in Mathematics, European Mathematical Society (EMS), Zürich, 2008. Translated, revised and expanded from the 2002 Russian original. MR2396717

[15] F. Bonahon, Bouts des variétés hyperboliques de dimension 3 (French), Ann. of Math. (2) 124 (1986), no. 1, 71-158, DOI 10.2307/1971388. MR847953

[16] B. H. Bowditch, A topological characterisation of hyperbolic groups, J. Amer. Math. Soc. 11 (1998), no. 3, 643-667, DOI 10.1090/S0894-0347-98-00264-1. MR1602069

[17] B. H. Bowditch, Convergence groups and configuration spaces, Geometric group theory down under (Canberra, 1996), de Gruyter, Berlin, 1999, pp. 23-54. MR.1714838

[18] B. H. Bowditch, The Cannon-Thurston map for punctured-surface groups, Math. Z. 255 (2007), no. 1, 35-76, DOI 10.1007/s00209-006-0012-4. MR2262721

[19] B. H. Bowditch, Stacks of hyperbolic spaces and ends of 3-manifolds, Geometry and topology down under, Contemp. Math., vol. 597, Amer. Math. Soc., Providence, RI, 2013, pp. 65-138, DOI 10.1090/conm/597/11769. MR.3186670

[20] M. R. Bridson and D. Groves, The quadratic isoperimetric inequality for mapping tori of free group automorphisms, Mem. Amer. Math. Soc. 203 (2010), no. 955, xii+152, DOI 10.1090/S0065-9266-09-00578-X. MR.2590896

[21] M. R. Bridson and A. Haefliger, Metric spaces of non-positive curvature, Grundlehren der Mathematischen Wissenschaften [Fundamental Principles of Mathematical Sciences], vol. 319, Springer-Verlag, Berlin, 1999. MR 1744486

[22] P. Brinkmann, Hyperbolic automorphisms of free groups, Geom. Funct. Anal. 10 (2000), no. 5, 1071-1089, DOI 10.1007/PL00001647. MR1800064 
[23] J. F. Brock, R. D. Canary, and Y. N. Minsky, The classification of Kleinian surface groups, II: The ending lamination conjecture, Ann. of Math. (2) 176 (2012), no. 1, 1-149, DOI 10.4007/annals.2012.176.1.1. MR2925381

[24] D. Calegari and D. Gabai, Shrinkwrapping and the taming of hyperbolic 3-manifolds, J. Amer. Math. Soc. 19 (2006), no. 2, 385-446, DOI 10.1090/S0894-0347-05-00513-8. MR2188131

[25] J. W. Cannon and W. P. Thurston, Group invariant Peano curves, Geom. Topol. 11 (2007), 1315-1355, DOI 10.2140/gt.2007.11.1315. MR2326947

[26] A. Casson and D. Jungreis, Convergence groups and Seifert fibered 3-manifolds, Invent. Math. 118 (1994), no. 3, 441-456, DOI 10.1007/BF01231540. MR.1296353

[27] A. J. Casson and S. A. Bleiler, Automorphisms of surfaces after Nielsen and Thurston, London Mathematical Society Student Texts, vol. 9, Cambridge University Press, Cambridge, 1988. MR 964685

[28] T. Coulbois and A. Hilion, Botany of irreducible automorphisms of free groups, Pacific J. Math. 256 (2012), no. 2, 291-307, DOI 10.2140/pjm.2012.256.291. MR.2944977

[29] T. Coulbois and A. Hilion, Rips induction: index of the dual lamination of an $\mathbb{R}$-tree, Groups Geom. Dyn. 8 (2014), no. 1, 97-134, DOI 10.4171/GGD/218. MR3209704

[30] T. Coulbois, A. Hilion, and M. Lustig, Non-unique ergodicity, observers' topology and the dual algebraic lamination for $\mathbb{R}$-trees, Illinois J. Math. 51 (2007), no. 3, 897-911. MR2379729

[31] T. Coulbois, A. Hilion, and M. Lustig, $\mathbb{R}$-trees and laminations for free groups. I. Algebraic laminations, J. Lond. Math. Soc. (2) 78 (2008), no. 3, 723-736, DOI 10.1112/jlms/jdn052. MR.2456901

[32] T. Coulbois, A. Hilion, and M. Lustig, $\mathbb{R}$-trees and laminations for free groups. II. The dual lamination of an $\mathbb{R}$-tree, J. Lond. Math. Soc. (2) 78 (2008), no. 3, 737-754, DOI 10.1112/jlms/jdn053. MR2456902

[33] S. Dowdall, I. Kapovich, and S. J. Taylor, Cannon-Thurston maps for hyperbolic free group extensions, Israel J. Math., to appear; arXiv:1506.06974

[34] S. Fenley, Ideal boundaries of pseudo-Anosov flows and uniform convergence groups with connections and applications to large scale geometry, Geom. Topol. 16 (2012), no. 1, 1-110, DOI 10.2140/gt.2012.16.1. MR.2872578

[35] W. J. Floyd, Group completions and limit sets of Kleinian groups, Invent. Math. 57 (1980), no. 3, 205-218, DOI 10.1007/BF01418926. MR568933

[36] E. M. Freden, Negatively curved groups have the convergence property. I, Ann. Acad. Sci. Fenn. Ser. A I Math. 20 (1995), no. 2, 333-348. MR1346817

[37] D. Gabai, Convergence groups are Fuchsian groups, Ann. of Math. (2) 136 (1992), no. 3, 447-510, DOI 10.2307/2946597. MR 1189862

[38] V. Gerasimov, Expansive convergence groups are relatively hyperbolic, Geom. Funct. Anal. 19 (2009), no. 1, 137-169, DOI 10.1007/s00039-009-0718-7. MR.2507221

[39] V. Gerasimov, Floyd maps for relatively hyperbolic groups, Geom. Funct. Anal. 22 (2012), no. 5, 1361-1399, DOI 10.1007/s00039-012-0175-6. MR2989436

[40] V. Gerasimov and L. Potyagailo, Quasi-isometric maps and Floyd boundaries of relatively hyperbolic groups, J. Eur. Math. Soc. (JEMS) 15 (2013), no. 6, 2115-2137, DOI 10.4171/JEMS/417. MR 3120738

[41] V. Gerasimov and L. Potyagailo, Similar relatively hyperbolic actions of a group, preprint, 2013; arXiv:1305.6649

[42] F. W. Gehring and G. J. Martin, Discrete quasiconformal groups. I, Proc. London Math. Soc. (3) 55 (1987), no. 2, 331-358, DOI 10.1093/plms/s3-55_2.331. MR896224

[43] M. Handel and L. Mosher, Axes in outer space, Mem. Amer. Math. Soc. 213 (2011), no. 1004, vi+104, DOI 10.1090/S0065-9266-2011-00620-9. MR2858636

[44] M. Handel and L. Mosher, Subgroup decomposition in Out $\left(F_{n}\right)$ : Introduction and Research Announcement, preprint, 2013; arXiv:1302.2681

[45] W. Jeon, I. Kim, K. Ohshika, and C. Lecuire, Primitive stable representations of free Kleinian groups, Israel J. Math. 199 (2014), no. 2, 841-866, DOI 10.1007/s11856-013-0062-3. MR.3219560

[46] W. Jeon and K. Ohshika, Measurable rigidity for Kleinian groups, Ergodic Theory and Dynamical Systems, to appear; published online June 2015; DOI: 10.1017/etds.2015.15

[47] I. Kapovich, A non-quasiconvexity embedding theorem for hyperbolic groups, Math. Proc. Cambridge Philos. Soc. 127 (1999), no. 3, 461-486, DOI 10.1017/S0305004199003862. MR 1713122 
[48] I. Kapovich, Algorithmic detectability of iwip automorphisms, Bull. Lond. Math. Soc. 46 (2014), no. 2, 279-290, DOI 10.1112/blms/bdt093. MR 3194747

[49] I. Kapovich and N. Benakli, Boundaries of hyperbolic groups, Combinatorial and geometric group theory (New York, 2000/Hoboken, NJ, 2001), Contemp. Math., vol. 296, Amer. Math. Soc., Providence, RI, 2002, pp. 39-93, DOI 10.1090/conm/296/05068. MR1921706

[50] I. Kapovich and M. Lustig, Geometric intersection number and analogues of the curve complex for free groups, Geom. Topol. 13 (2009), no. 3, 1805-1833, DOI 10.2140/gt.2009.13.1805. MR2496058

[51] I. Kapovich and M. Lustig, Intersection form, laminations and currents on free groups, Geom. Funct. Anal. 19 (2010), no. 5, 1426-1467, DOI 10.1007/s00039-009-0041-3. MR2585579

[52] I. Kapovich and M. Lustig, Invariant laminations for irreducible automorphisms of free groups, Q. J. Math. 65 (2014), no. 4, 1241-1275, DOI 10.1093/qmath/hat056. MR 3285770

[53] I. Kapovich and M. Lustig, Cannon-Thurston fibers for iwip automorphisms of $F_{N}$, J. Lond. Math. Soc. (2) 91 (2015), no. 1, 203-224, DOI 10.1112/jlms/jdu069. MR.3335244

[54] I. Kapovich and A. Myasnikov, Stallings foldings and subgroups of free groups, J. Algebra 248 (2002), no. 2, 608-668, DOI 10.1006/jabr.2001.9033. MR.1882114

[55] I. Kapovich and H. Short, Greenberg's theorem for quasiconvex subgroups of word hyperbolic groups, Canad. J. Math. 48 (1996), no. 6, 1224-1244, DOI 10.4153/CJM-1996-065-6. MR 1426902

[56] M. Kapovich, On the absence of Sullivan's cusp finiteness theorem in higher dimensions, Algebra and analysis (Irkutsk, 1989), Amer. Math. Soc. Transl. Ser. 2, vol. 163, Amer. Math. Soc., Providence, RI, 1995, pp. 77-89. MR.1331386

[57] M. Kapovich and B. Kleiner, Hyperbolic groups with low-dimensional boundary (English, with English and French summaries), Ann. Sci. École Norm. Sup. (4) 33 (2000), no. 5, 647-669, DOI 10.1016/S0012-9593(00)01049-1. MR.1834498

[58] R. P. Kent IV and C. J. Leininger, Shadows of mapping class groups: capturing convex cocompactness, Geom. Funct. Anal. 18 (2008), no. 4, 1270-1325, DOI 10.1007/s00039-0080680-9. MR2465691

[59] R. P. Kent IV and C. J. Leininger, Uniform convergence in the mapping class group, Ergodic Theory Dynam. Systems 28 (2008), no. 4, 1177-1195, DOI 10.1017/S0143385707000818. MR2437226

[60] E. Klarreich, Semiconjugacies between Kleinian group actions on the Riemann sphere, Amer. J. Math. 121 (1999), no. 5, 1031-1078. MR1713300

[61] C. Leininger, D. D. Long, and A. W. Reid, Commensurators of finitely generated nonfree Kleinian groups, Algebr. Geom. Topol. 11 (2011), no. 1, 605-624, DOI 10.2140/agt.2011.11.605. MR.2783240

[62] C. J. Leininger, M. Mj, and S. Schleimer, The universal Cannon-Thurston map and the boundary of the curve complex, Comment. Math. Helv. 86 (2011), no. 4, 769-816, DOI 10.4171/CMH/240. MR2851869

[63] G. Levitt and M. Lustig, Irreducible automorphisms of $F_{n}$ have north-south dynamics on compactified outer space, J. Inst. Math. Jussieu 2 (2003), no. 1, 59-72, DOI 10.1017/S1474748003000033. MR 1955207

[64] C. T. McMullen, Local connectivity, Kleinian groups and geodesics on the blowup of the torus, Invent. Math. 146 (2001), no. 1, 35-91, DOI 10.1007/PL00005809. MR.1859018

[65] Y. N. Minsky, On rigidity, limit sets, and end invariants of hyperbolic 3-manifolds, J. Amer. Math. Soc. 7 (1994), no. 3, 539-588, DOI 10.2307/2152785. MR.1257060

[66] Y. Minsky, The classification of Kleinian surface groups. I. Models and bounds, Ann. of Math. (2) 171 (2010), no. 1, 1-107, DOI 10.4007/annals.2010.171.1. MR2630036

[67] M. Mitra, Ending laminations for hyperbolic group extensions, Geom. Funct. Anal. 7 (1997), no. 2, 379-402, DOI 10.1007/PL00001624. MR.1445392

[68] M. Mitra, Cannon-Thurston maps for hyperbolic group extensions, Topology 37 (1998), no. 3, 527-538, DOI 10.1016/S0040-9383(97)00036-0. MR1604882

[69] M. Mitra, Cannon-Thurston maps for trees of hyperbolic metric spaces, J. Differential Geom. 48 (1998), no. 1, 135-164. MR 1622603

[70] H. Miyachi, Semiconjugacies between actions of topologically tame Kleinian groups, preprint, 2002 . 
[71] M. Mj, Ending laminations and Cannon-Thurston maps, Geom. Funct. Anal. 24 (2014), no. 1, 297-321, DOI 10.1007/s00039-014-0263-x. With an appendix by Shubhabrata Das and Mj. MR3177384

[72] M. Mj, Cannon-Thurston maps for pared manifolds of bounded geometry, Geom. Topol. 13 (2009), no. 1, 189-245, DOI 10.2140/gt.2009.13.189. MR2469517

[73] M. Mj, Cannon-Thurston maps, i-bounded geometry and a theorem of McMullen, Actes du Séminaire de Théorie Spectrale et Géometrie. Volume 28. Année 2009-2010, Sémin. Théor. Spectr. Géom., vol. 28, Univ. Grenoble I, Saint-Martin-d'Hères, 2010, pp. 63-107, DOI 10.5802/tsg.279. MR2848212

[74] M. Mj, Cannon-Thurston maps and bounded geometry, Teichmüller theory and moduli problem, Ramanujan Math. Soc. Lect. Notes Ser., vol. 10, Ramanujan Math. Soc., Mysore, 2010, pp. 489-511. MR2667569

[75] M. Mj, Cannon-Thurston Maps for Kleinian Groups, arXiv:1002.0996

[76] M. Mj, On discreteness of commensurators, Geom. Topol. 15 (2011), no. 1, 331-350, DOI 10.2140/gt.2011.15.331. MR.2776846

[77] M. Mj, Cannon-Thurston maps for surface groups, Ann. of Math. (2) 179 (2014), no. 1, 1-80, DOI 10.4007/annals.2014.179.1.1. MR3126566

[78] M. Mj and A. Pal, Relative hyperbolicity, trees of spaces and Cannon-Thurston maps, Geom. Dedicata 151 (2011), 59-78, DOI 10.1007/s10711-010-9519-2. MR2780738

[79] M. Queffélec, Substitution dynamical systems - spectral analysis, 2nd ed., Lecture Notes in Mathematics, vol. 1294, Springer-Verlag, Berlin, 2010. MR2590264

[80] I. Rivin, Zariski density and genericity, Int. Math. Res. Not. IMRN 19 (2010), 3649-3657, DOI 10.1093/imrn/rnq043. MR2725508

[81] J. Souto, Cannon-Thurston maps for thick free groups, preprint, 2006. http://www.math.ubc.ca/ jsouto/papers/Cannon-Thurston.pdf.

[82] D. Sullivan, Discrete conformal groups and measurable dynamics, Bull. Amer. Math. Soc. (N.S.) 6 (1982), no. 1, 57-73, DOI 10.1090/S0273-0979-1982-14966-7. MR634434

[83] E. L. Swenson, Quasi-convex groups of isometries of negatively curved spaces, Topology Appl. 110 (2001), no. 1, 119-129, DOI 10.1016/S0166-8641(99)00166-2. Geometric topology and geometric group theory (Milwaukee, WI, 1997). MR.1804703

[84] P. Tukia, A rigidity theorem for Möbius groups, Invent. Math. 97 (1989), no. 2, 405-431, DOI 10.1007/BF01389048. MR1001847

[85] P. Tukia, Convergence groups and Gromov's metric hyperbolic spaces, New Zealand J. Math. 23 (1994), no. 2, 157-187. MR 1313451

[86] P. Tukia, Conical limit points and uniform convergence groups, J. Reine Angew. Math. 501 (1998), 71-98, DOI 10.1515/crll.1998.081. MR.1637829

[87] W. P. Thurston, The geometry and topology of three-manifolds, Lecture Notes from Princeton University, 1978-1980.

[88] A. Yaman, A topological characterisation of relatively hyperbolic groups, J. Reine Angew. Math. 566 (2004), 41-89, DOI 10.1515/crll.2004.007. MR.2039323

School of Mathematics, KiAS, Hoegiro 87, Dongdaemun-gu, Seoul, 130-722, Korea

E-mail address: jwoojin@kias.re.kr

Department of Mathematics, University of Illinois at Urbana-Champaign, 1409 West

Green Street, Urbana, Illinois 61801

URL: http://www.math.uiuc.edu/ kapovich/

E-mail address: kapovich@math.uiuc.edu

Department of Mathematics, University of Illinois at Urbana-Champaign, 1409 West

Green Street, Urbana, Illinois 61801

URL: http://www.math.uiuc.edu/ clein/

E-mail address: clein@math.uiuc.edu

Department of Mathematics, Graduate School of Science, Osaka University, ToyONAKA, OSAKA 560-0043, JAPAN

E-mail address: ohshika@math.sci.osaka-u.ac.jp 